\title{
LOW REGULARITY SOLUTIONS FOR THE WAVE MAP EQUATION INTO THE 2-D SPHERE
}

\author{
PIERO D'ANCONA AND VLADIMIR GEORGIEV
}

\begin{abstract}
A class of weak wave map solutions with initial data in Sobolev space of order $s<1$ is studied. A non uniqueness result is proved for the case, when the target manifold is a two dimensional sphere. Using an equivariant wave map ansatz a family of self - similar solutions is constructed. This construction enables one to show ill - posedness of the inhomogeneous Cauchy problem for wave maps.
\end{abstract}

\section{INTRODUCTION}

Let $(M, \eta)$ be a pseudoriemannian manifold and $(N, g)$ be a riemannian manifold. For smooth maps $u: M \rightarrow N$ we can compute the Lagrangean density $L(u)=$ $\operatorname{Tr}_{\eta}\left(u^{*} g\right)$, i.e., the trace with respect to the metric $\eta$ of the pullback through $u$ of the metric $g$. In local coordinates $\left(x^{\alpha}\right)$ on $M$ and $\left(u^{a}\right)$ on $N$ we can write

$$
L(u)=\eta^{\alpha \beta} g_{a b} \frac{\partial u^{a}}{\partial x^{\alpha}} \frac{\partial u^{b}}{\partial x^{\beta}}
$$

where we use the summation convention over repeated indices. A stationary map for $L$ is called a wave map, $M$ is called the base manifold and $N$ the target manifold. Wave maps arise in different physical theories and are the object of active investigation (see e.g. [2], [3], [5], [6], [7], [8], [9], [10], [12], [14], [16], [18], [20]; see also the book [13] and the refences therein).

For simplicity, it is customary to consider the case of a flat Minkowsky $M$, that is to say $M=\mathbb{R} \times \mathbb{R}^{n}$ with metric

$$
\eta^{\alpha \beta}=\operatorname{diag}[-1,1, \ldots, 1] .
$$

Even in this situation most of the essential difficulties of the problem are still present. Notice that, at least in the case of compact manifolds, by Nash' theorem it is not restrictive to assume that $N$ is embedded isometrically in some $\mathbb{R}^{d}$ with $d$ larger than the dimension $k$ of $N$. Writing $\partial_{\alpha}=\partial / \partial x^{\alpha}, \partial^{\alpha}=\eta^{\alpha \beta} \partial_{\beta}$, we are thus interested in the stationary points of the functional

$$
\mathcal{L}(u)=\int_{\mathbb{R} \times \mathbb{R}^{n}} g_{a b}(u) \partial^{\alpha} u^{a} \partial_{\alpha} u^{b} d x^{0} \ldots d x^{n} .
$$

The corresponding Euler-Lagrange equations are

$$
-2 \partial_{\alpha}\left(g_{a b}(u) \partial^{\alpha} u^{b}\right)+\partial_{\alpha} u^{c} \partial^{\alpha} u^{b} \partial_{a} g_{b c}=0
$$

or equivalently

$$
g_{a b} \square u^{b}+\partial_{c} g_{a b} \partial_{\alpha} u^{c} \partial^{\alpha} u^{b}-\frac{1}{2} \partial_{a} g_{b c} \partial_{\alpha} u^{c} \partial^{\alpha} u^{b}=0
$$

Key words and phrases. AMS Subject Classification: 35L70, 58J45, . Keywords: Wave map, Cauchy problem, nonlinear equation, hyperbolic equation, ill-posed problem. 
where $\square=-\partial_{\alpha} \partial^{\alpha}=\partial_{0}^{2}-\partial_{1}^{2}-\cdots-\partial_{n}^{2}$. Writing the second sum in the following form

$$
\partial_{c} g_{a b} \partial_{\alpha} u^{c} \partial^{\alpha} u^{b}=\frac{1}{2}\left(\partial_{c} g_{b a}+\partial_{b} g_{a c}\right) \partial_{\alpha} u^{c} \partial^{\alpha} u^{b}
$$

and recalling the definition of the Christoffel symbols

$$
\Gamma_{a ; b c}=\frac{1}{2}\left(\partial_{b} g_{a, c}+\partial_{c} g_{a, b}-\partial_{a} g_{b, c}\right), \quad \Gamma_{b c}^{a}=g^{a i} \Gamma_{i ; b c}
$$

we obtain

$$
g_{a b} \square u^{b}+\Gamma_{a ; b c} \partial_{\alpha} u^{c} \partial^{\alpha} u^{b}=0
$$

and finally

$$
\square u^{\ell}+\Gamma_{b c}^{\ell}(u) \partial_{\alpha} u^{b} \partial^{\alpha} u^{c}=0 .
$$

This is the wave map system in local coordinates on $N$, which is hyperbolic in the direction of $t=x_{0}$. Thus the natural problem for (1.4) is the Cauchy problem, with initial data at $t=0$

$$
u(0, x)=u_{0}(x), \quad u_{t}(0, x)=u_{1}(x) .
$$

When $N$ is the unit sphere in $\mathbb{R}^{d}$ with the metric induced by the Euclidean metric, if we choose as a chart for the upper (or the lower) half sphere $u_{d}>0$ the projection on $\left(u_{1}, \ldots, u_{d-1}\right)$, a direct computation gives $\Gamma_{i j}^{\ell}(u)=u^{\ell} \delta_{i j}+u^{i} u^{j} u^{\ell} /\left(u^{d}\right)^{2}$; the equations become very symmetrical using the full set of functions $\left(u_{1}, \ldots, u_{d}\right)$, indeed we obtain in a few steps

$$
\square u+\left(\left|u_{t}\right|^{2}-\left|\nabla_{x} u\right|^{2}\right) u=0
$$

subject to the constraint $|u|=1$. It is easy to see that if a smooth function $u(t, x)$ solves (1.4) and moreover satisfies the constraint at $t=0$, i.e. $|u(0, x)|=1$, $u(0, x) \cdot u_{t}(0, x)=0$, then we have $|u|=1$ for all $t$. It is also possible to consider system (1.4) without geometric constrains, from a purely analytical point of view.

The minimal regularity required of $u$ in order to give a meaning to the nonlinear term (in distributional sense) is $D_{t, x} u \in L_{\text {loc }}^{2}$, provided $|u|=1$. This can be further relaxed if we remark that $\square u$ is parallel to $u$ by the equation, hence we have also

$$
u \wedge \square u=0, \quad|u|=1
$$

or equivalently

$$
\partial_{t}\left(u_{t} \wedge u\right)=\sum_{j=1}^{n} \partial_{x_{j}}\left(u_{x_{j}} \wedge u\right),
$$

since $u \cdot u_{t}=u \cdot u_{x_{j}}=0$ by the condition $|u|=1$. For smooth functions with norm $1,(1.5)$ and (1.4) are equivalent; moreover, equation (1.5) has a distributional sense for $D u \in L_{\text {loc }}^{1}$ only, provided $|u|=1$. Thus in the following we shall say that $u$ is a weak solution of equation (1.4) if $D u \in L_{\mathrm{loc}}^{1},|u|=1$ a.e. and $u$ satisfies (1.5) in the sense of distributions.

One of the basic open questions of the theory is the well posedness of the Cauchy problem for (1.4) in two dimensions, i.e. with

$$
u: \mathbb{R} \times \mathbb{R}^{2} \rightarrow \mathbb{S}^{2} \subseteq \mathbb{R}^{3} .
$$

In this case the local existence of smooth solutions follows by classical arguments, while global existence meets essential difficulties. The critical space for equation (1.5) in two dimensions is $H^{1}$, which is also the energy space; thus an important question is the well posedness of (1.5) in $H^{1}$.

The aim of this paper is to investigate the behaviour of (1.4) for solutions of low regularity, i.e., below the energy space $H^{1} \times L^{2}$, and indeed to show that in general the problem is not well posed in this situation.

Our first result is the following: 
NonUNiQUENESS [Theorems 3.1 and 3.2]. It is possible to construct two weak solutions $u, v: \mathbb{R} \times \mathbb{R}^{2} \rightarrow \mathbb{S}^{2}$ to equation (1.4), continuous in time with values in $H^{1-\varepsilon}(\Omega)$ for all $\varepsilon>0$ and all bounded $\Omega$, and also $L^{\infty}$ with values in the Besov space $B_{2, \infty}^{1}$, such that $u \equiv v$ for $t<0$ and $u \not \equiv v$ for $t>0$. We can construct $u, v$ such that their Besov norm is arbitrarily small.

Since $H^{1}=B_{2,2}^{1}$, recalling Tataru's local well posedness result in $B_{2,1}^{1}$, we may say that the question of local well posedness in $H^{1}$ is confined in the gap between the two Besov spaces $B_{2, \infty}^{1}$ and $B_{2,1}^{1}$. In this regard it is necessary to mention Tao's result [17] showing that the well posedness holds provided the data are slightly smoother $\left(H^{1+\varepsilon} \times H^{\varepsilon}\right)$ and small in the $\dot{H}^{1}$ norm. See also the paper [11], where a model (scalar) equation with the same type of nonlinearity is considered.

Our second result concerns stability of (1.4) in presence of a forcing term, namely we consider the following Cauchy problem

$$
\begin{aligned}
& \square u+\left(\left|u_{t}\right|^{2}-\left|\nabla_{x} u\right|^{2}\right) u=F(t, x), \quad u: \mathbb{R} \times \mathbb{R}^{2} \rightarrow \mathbb{S}^{2} \\
& u(0, x)=0, \quad u_{t}(0, x)=0
\end{aligned}
$$

and we prove the following result:

Ill-Posedness [Theorem 4.1]. Denote by $N=(0,0,1)$ the North pole. We can construct a sequence of functions $F=F_{k}$ with support in the forward light cone such that (1.6), (1.7) has a solution $u_{k}$ with $u_{k}-N \in C\left(\mathbb{R} ; H^{1+\varepsilon}\left(\mathbb{R}^{2}\right),\left(\varepsilon=\varepsilon_{k} \rightarrow 0\right)\right.$, such that $u_{k}(0, x)=N, \partial_{t} u_{k}(0, X)=0$ for all $k$. Moreover, for all $T>0$ the $H^{1}\left(\mathbb{R}^{2}\right)$ norm of $u_{k}-N$ at $t=T$ diverges to $+\infty$ while the norm of $F_{k}$ in $L^{p}\left([0, T], L^{q}\left(\mathbb{R}^{2}\right)\right)$ tends to 0 as $k \rightarrow \infty$, provided $p \geq 1$, $q \geq 2$ satisfy

In other words, the solution map

$$
\frac{1}{p}+\frac{2}{q}>2
$$

$$
\left(u_{0}, u_{1}, F\right) \mapsto u(t, x)
$$

between the spaces

$$
H^{1} \times L^{2} \times L^{p} L^{q} \rightarrow C H^{1}, \quad \frac{1}{p}+\frac{2}{q}>2
$$

is not continuous at $(N, 0,0)$. Notice that the "correct" energy space for the forcing term is $L^{1} L^{2}$, and $p=1, q=2$ do not satisfy the condition $1 / p+2 / q>2$. Thus also our second result applies only in the low regularity case.

Clearly, well posedness for (1.6) is a more general problem than for the homogeneous equation (1.4). At least, we can say that the above result rules out existence proofs for (1.4) based on contraction methods in the said spaces. Indeed, such methods would imply that the solution map data $\mapsto$ solution is analytic, while the above result shows that the map is not even locally bounded. We also mention that even in the homogeneous case and in the critical space it is possible to prove that the solution map is not smooth, actually not uniformly continuous; for a precise proof we refer to the forthcoming paper [4].

The plan of the paper is the following: in Section 2 we recall the definition of equivariant and self-similar solutions, which are necessary for the following constructions. The self-similar Ansatz leads to an ODE which is studied in detail in Appendix A; in particular all the solutions are computed explicitly. Section 3 is devoted to the non uniqueness result, while the ill posedness of the Cauchy problem with a forcing term is proved in Section 4. Appendix B collects some technical lemmas. 


\section{EQUIVARIANT AND SELF-SIMILAR SOLUTIONS}

When the manifolds have rotational symmetry, two interesting classes of special solutions arise, the equivariant and the self-similar solutions. The general construction is standard and can be found in [1], [13], [14].

We recall briefly the equivariant Anzatz. Assume that $N$ is a smooth $\ell$-dimensional rotationally symmetric manifold defined as

$$
N=\left\{(\phi, \chi) ; \phi \in\left[0, \phi^{*}\right), \chi \in \mathbb{S}^{\ell-1}\right\}
$$

$\left(\phi^{*}\right.$ may be $\left.+\infty\right)$, with metric

$$
d \phi^{2}+g(\phi)^{2} d \chi^{2}
$$

where $d \chi^{2}$ is the standard metric on $\mathbf{S}^{\ell-1}$. In the coordinates $\left(\phi ; \chi_{1}, \ldots, \chi_{\ell-1}\right)$, denoting by $h_{i j}$ the coefficients of the metric $d \chi^{2}$, the only nonzero Christoffel symbols for $(2.1)$ are

$$
\Gamma_{\chi_{i} \chi_{j}}^{\phi}=-g^{\prime}(\phi) g(\phi) h_{i j}, \quad \Gamma_{\chi_{j} \phi}^{\chi_{i}}=\frac{g^{\prime}(\phi)}{g(\phi)} \delta_{i j}, \quad \Gamma_{\chi_{j} \chi_{s}}^{\chi_{i}}=\gamma_{j s}^{i},
$$

where $\gamma_{j s}^{i}$ are the Christoffel symbols for the metric $h_{i j}$. The equivariant wave maps are the maps $u$ satisfying the Ansatz

$$
u(t, x)=(\phi, \chi), \quad \phi=\phi(t, r), \quad \chi=\chi(\omega),
$$

where $(r, \omega)$ are the spherical coordinates on $\mathbb{R}^{n}$. Under this assumption, the equations for $\chi$ decouple and in fact we obtain that $\chi: \mathbb{S}^{n-1} \rightarrow \mathbb{S}^{\ell-1}$ must be a harmonic map. For suitable choices of the dimensions $n, \ell$ (and of $\chi$ ) further simplifications occur. In the special case $\ell=n=2$ under consideration here, it is easy to see that $\chi$ must be a rotation of degree $k=1,2,3, \ldots$ of $\mathbb{S}^{1}$ into itself. With this choice the equation for $\phi$ decouples and we obtain

$$
\phi_{t t}-\phi_{r r}-\frac{1}{r} \phi_{r}+\frac{k^{2}}{r^{2}} g^{\prime}(\phi) g(\phi)=0 .
$$

This is the equivariant wave map equation.

When $N$ is the sphere $\mathbb{S}^{2}$, the above framework corresponds to the standard choice of coordinates $(\phi, \chi)$ with $\phi \in[0, \pi]$ and $\chi \in \mathbb{S}^{1}$. Then the metric can be written

and equation (2.4) becomes

$$
d \phi^{2}+\sin ^{2} \phi d \chi^{2}
$$

$$
\phi_{t t}-\phi_{r r}-\frac{1}{r} \phi_{r}+\frac{k^{2}}{2 r^{2}} \sin (2 \phi)=0 .
$$

Actually it is more convenient to embed $N=\mathbb{S}^{2}$ in $\mathbb{R}^{3}$; with the usual coordinate system on $N$

$$
\left(\begin{array}{c}
\sin \phi \sin \chi \\
\sin \phi \sin \chi \\
\cos \phi
\end{array}\right)
$$

where $\phi \in[0, \pi], \chi \in[0,2 \pi], \phi=0$ corresponding to the north pole and $\phi=\pi$ to the south pole of the unit sphere. Then we can express the solution $u(t, x)$ to $(1.4)$ as the vector $\left(u_{1}, u_{2}, u_{3}\right)$ with

$$
\begin{aligned}
& u_{1}=\sin \phi(t,|x|) \cdot|x|^{-k} \operatorname{Re}\left(x_{1}+i x_{2}\right)^{k} \\
& u_{2}=\sin \phi(t,|x|) \cdot|x|^{-k} \operatorname{Im}\left(x_{1}+i x_{2}\right)^{k} \\
& u_{3}=\cos \phi(t,|x|) ;
\end{aligned}
$$

since $\cos \chi=\cos (k \omega)=|x|^{-k} \operatorname{Re}\left(x_{1}+i x_{2}\right)^{k}, \sin \chi=\sin (k \omega)=|x|^{-k} \operatorname{Im}\left(x_{1}+\right.$ $\left.i x_{2}\right)^{k}$. 
In order to introduce the self-similar solutions, we recast the equation in the hyperbolic coordinates

$$
\rho=\sqrt{t^{2}-r^{2}}, \quad \tau=\frac{r}{t}
$$

notice that the inverse transformations are given by

$$
t=\frac{\rho}{\sqrt{1-\tau^{2}}}, \quad r=\frac{\tau \rho}{\sqrt{1-\tau^{2}}} .
$$

Then we obtain

$$
\partial_{t}^{2}-\partial_{r}^{2}-\frac{1}{r} \partial_{r}=\partial_{\rho}^{2}+\frac{2}{\rho} \partial_{\rho}-\frac{\Delta_{H}}{\rho^{2}}
$$

where $\Delta_{H}$ is the Laplace operator on the hyperboloid $\rho=1$

$$
\Delta_{H}=\left(1-\tau^{2}\right)^{2} \partial_{\tau}^{2}-\frac{\left(1-\tau^{2}\right)\left(2 \tau^{2}-1\right)}{\tau} \partial_{\tau} .
$$

In the new coordinates $\rho, \tau$ the equation (2.4) becomes

$$
\partial_{\rho}^{2} \phi+\frac{2}{\rho} \partial_{\rho} \phi-\frac{1}{\rho^{2}} \Delta_{H} \phi+k^{2} \frac{\left(1-\tau^{2}\right)}{2 \tau^{2} \rho^{2}} \sin 2 \phi=0 .
$$

We can now define the self-similar solutions as the solutions which are independent of $\rho$, i.e.,

$$
\phi(t, r)=\psi\left(\frac{r}{t}\right) \equiv \psi(\tau) .
$$

Under this assumption the first two terms in (2.10) drop, and we obtain immediately the following equation for $\psi=\psi(\tau)$ :

$$
\tau^{2}\left(\tau^{2}-1\right) \psi^{\prime \prime}+\tau\left(2 \tau^{2}-1\right) \psi^{\prime}+k^{2} \sin \psi \cos \psi=0
$$

Notice that if one could find global smooth solutions to this equation, an immediate consequence would be a blow up result for the wave map equation (1.4); but this is not possible, as shown in [13] (while in higher dimensions this idea is correct and was exploited in [14]). Nevertheless it is possible to utilize the singular (i.e., not in $H^{1}$ ) solutions thus obtained, as we shall do in the following section.

It is not difficult to see that all solutions to (2.11) are analytic and defined for $\tau \neq 0,1$; in Appendix A we give a complete study of the equation, and we represent all its solutions using Jacobi's elliptic functions. In the next sections, in particular, we shall use the following special solutions:

(i) The function defined as

$$
\psi(\tau)= \begin{cases}\arcsin \tau & \text { for } 0 \leq \tau \leq 1 \\ \pi / 2 & \text { for } \tau>1\end{cases}
$$

is a solution to the equation (for $\tau \neq 1$ ) in the case $k=1$. Notice that the only constant solutions are the integer multiples of $\pi / 2$, and that the value of the constant chosen here for $\tau>1$ ensures (Hölder) continuity. The fact that (2.12) is a solution can be verified directly, or can be obtained by setting $\lambda=\pi / 2, k=1$ in the general expression (5.26).

(ii) A more general class of solutions in the case $k=1$ is given by the expressions $(\delta \in] 0,1[)$

$$
\psi(\tau)= \begin{cases}2 \arctan \left[\tan \frac{\lambda}{2} \cdot \frac{\tau}{1 \pm \sqrt{1-\tau^{2}}}\right] & \text { for } 1 \leq \tau \leq 2 \\ \operatorname{am}\left(\delta \arctan \frac{1}{\sqrt{\tau^{2}-1}} \mid \frac{1}{\delta}\right) & \text { for } \tau>1 .\end{cases}
$$

Here the two-parameter function am $(\tau \mid m)$ is the Jacobi amplitude in the case $m>1$; a precise definition is given in Appendix A, here we shall only need to know 
that it is an analytic periodic function of $\tau$, with the property am $(0 \mid m)=0$, hence (2.13) tends to 0 as $\tau \rightarrow \infty$. Again, we can ensure Hölder continuity at $\tau=1$ by imposing a suitable condition on the constants $\lambda, \delta$. In the following section we shall also need to compute $\sin \psi$ and $\cos \psi$ for $\psi$ given by (2.13); writing

$$
\gamma=\tan \frac{\lambda}{2}
$$

we have

$$
\sin \psi(\tau)= \begin{cases}\frac{4 \gamma \tau}{\left(1 \pm \sqrt{1-\tau^{2}}\right)+\gamma^{2}\left(1 \mp \sqrt{1-\tau^{2}}\right)} & \text { for } 0 \leq \tau \leq 1 \\ \delta \cdot \operatorname{sn}\left(\arctan \frac{1}{\sqrt{\tau^{2}-1}} \mid \delta\right) & \text { for } \tau>1 .\end{cases}
$$

and

$$
\cos \psi(\tau)= \begin{cases}\frac{\left(1 \pm \sqrt{1-\tau^{2}}\right)-\gamma^{2}\left(1 \mp \sqrt{1-\tau^{2}}\right)}{\left(1 \pm \sqrt{1-\tau^{2}}\right)+\gamma^{2}\left(1 \mp \sqrt{1-\tau^{2}}\right)} & \text { for } 0 \leq \tau \leq 1 \\ \ln \left(\arctan \frac{1}{\sqrt{\tau^{2}-1}} \mid \delta\right), & \text { for } \tau>1\end{cases}
$$

(the signs \pm are the same as in (2.13), the $\mp$ are opposite). For the definition and properties of the elliptic functions sn $(\tau \mid \delta), \operatorname{dn}(\tau \mid \delta)$ see Appendix A. These formulas are proved in Appendix A, see Remark 5.2 and (5.29), (5.30), (5.31).

\section{LOW REGULARITY SELF-SIMILAR SOLUTIONS}

Our purpose here is to construct weak solutions to the wave map equation (1.4) from $\mathbb{R} \times \mathbb{R}^{2}$ to $\mathbb{S}^{2}$ below critical regularity. As mentioned in the Introduction, it is convenient to transform the equation in the form of a conservation law

$$
\partial_{t}\left(\partial_{t} u \wedge u\right)=\sum_{j=1}^{2} \partial_{j}\left(\partial_{j} u \wedge u\right)
$$

in order to handle weak solutions of very low regularity; indeed if $u$ is a locally bounded function such that $\partial u \in L_{\text {loc }}^{1}\left(\mathbb{R} \times \mathbb{R}^{2}\right)$, all the terms in (3.1) have a welldefined meaning in distribution sense.

Starting from a solution $\psi(\tau)$ of $(2.11)$, we can construct a self-similar solution to the wave map equation by setting

$$
u(t, x)=\left(\begin{array}{c}
u_{1}(t, x) \\
u_{2}(t, x) \\
u_{9}(t, x)
\end{array}\right),
$$

with

$$
\begin{aligned}
& u_{1}(t, x)=|x|^{-k} \operatorname{Re}\left(x_{1}+i x_{2}\right)^{k} \sin (\psi(|x| / t)) \\
& u_{2}(t, x)=|x|^{-k} \operatorname{Im}\left(x_{1}+i x_{2}\right)^{k} \sin (\psi(|x| / t)) \\
& u_{3}(t, x)=\cos (\psi(|x| / t)) .
\end{aligned}
$$

We shall restrict ourselves to the case $k=1$ and the special solutions examined in the preceding section. Our first result is based on the solution (2.13); this means simply

$$
u(t, x)=\frac{1}{t}\left(\begin{array}{c}
x_{1} \\
x_{2} \\
\sqrt{t^{2}-|x|^{2}}
\end{array}\right)
$$


inside the cone, and

$$
u(t, x)=\left(\begin{array}{c}
x_{1} /|x| \\
x_{2} /|x| \\
0
\end{array}\right)
$$

outside the cone.

Our aim is to prove

Theorem 3.1. The function $u(t, x)$ defined in (3.3), (3.4) is a solution in distribution sense of the wave map equation (3.1). Moreover, given any bounded open set $\Omega \subseteq \mathbb{R}^{2}$, we have

$$
u \in C\left(R, H^{s}(\Omega)\right), \quad \forall s<1 \quad \text { and } \quad u \in L^{\infty}\left(R, B_{2, \infty}^{1}(\Omega)\right) .
$$

$A$ second solution is the function $v(t, x)$, independent of $t$, defined as (3.4) everywhere; we have $v \in C\left(R, B_{2, \infty}^{1}(\Omega)\right)$ for any $\Omega$, and $v \equiv u$ for $t \leq 0$, thus the weak solution to (3.1) is not unique in the spaces (3.5).

Remark 3.1. Actually the solution $u$ is continuous with values in Besov for $t \geq 0$ and $t \leq 0$, and has a jump at $t=0$ only. We also notice that, thanks to the scaling properties of Besov spaces, the $\dot{B}_{2, \infty}^{1}$ norm of $u(t, x)$ on the ball $B(0, t)$ is constant as $t \downarrow 0$, i.e., it concentrates at 0 .

Proof. We remark that the first derivatives of $u$ in distribution sense coincide with the derivatives a.e., which are locally integrable functions on $\mathbb{R} \times \mathbb{R}^{2}$. This follows at once by Lemma 6.3 in the Appendix (with obvious choices); indeed, the singularities of $u$ are concentrated along the positive cone $t=|x|$, where $u$ is Hölder continuous, and along the line $x=0$ for negative $t$, where it is bounded. Thus we can compute the derivatives $u_{t}, u_{x_{j}}$ directly by differentiating the above formulas, obtaining locally integrable functions. A similar remark holds for $v$.

Outside the positive light cone, i.e. for $t \leq|x|$ (including negative $t$ ) we have $u \equiv v$, and an explicit computation gives

$$
\begin{gathered}
\partial_{t} u \wedge u=\partial_{t} v \wedge v=0 \\
\partial_{x_{1}} u \wedge u=\partial_{x_{1}} v \wedge v=\left(\begin{array}{c}
0 \\
0 \\
x_{2} /|x|^{2}
\end{array}\right), \\
\partial_{x_{2}} u \wedge u=\partial_{x_{2}} v \wedge v=\left(\begin{array}{c}
0 \\
0 \\
-x_{1} /|x|^{2}
\end{array}\right) .
\end{gathered}
$$

These formulas hold on the whole space $\mathbb{R} \times \mathbb{R}^{2}$ for the second solution $v$.

To check that $v$ is a solution of (3.1) we may proceed directly; indeed, we have to check that

$$
\partial_{x_{1}}\left[-\frac{x_{2}}{|x|^{2}}\right]+\partial_{x_{2}}\left[\frac{x_{1}}{|x|^{2}}\right]=0
$$

in distribution sense, and this follows from the identities

$$
\partial_{x_{j}}|x|=\frac{x_{j}}{|x|}
$$

(notice that these functions are locally integrable).

To check that $u$ is a solution, it is simpler to express the equation in spherical coordinates:

$$
\partial_{t}\left(u_{t} \wedge u\right)-\partial_{r}\left(u_{r} \wedge u\right)-\frac{1}{r} u_{r} \wedge u-\frac{1}{r^{2}} \partial_{\omega}\left(\partial_{\omega} u \wedge u\right)=0 .
$$


Outside the light cone we have

$$
u=(\cos \omega, \sin \omega, 0)
$$

while inside it

$$
u=\frac{1}{t}\left(r \cos \omega, r \sin \omega, \sqrt{t^{2}-r^{2}}\right) .
$$

We can write the wedge products in a global form valid everywhere: writing for brevity

$$
Z(\omega)=\left(\begin{array}{c}
-\sin \omega \\
\cos \omega \\
0
\end{array}\right)
$$

and

$$
w(t, r)= \begin{cases}\frac{1}{\sqrt{t^{2}-r^{2}}} & \text { if } r \leq t \\ 0 & \text { otherwise }\end{cases}
$$

we find after some computations

$$
\begin{aligned}
& u_{t} \wedge u=\frac{r}{t} w(t, r) \cdot Z, \\
& u_{r} \wedge u=-w(t, r) \cdot Z,
\end{aligned}
$$

while

$$
\partial_{\omega}\left(\partial_{\omega} u \wedge u\right)=\Delta_{\omega} u \wedge u=\frac{r}{t^{2}} \sqrt{t^{2}-r^{2}} \cdot Z .
$$

Thus, to check that $u$ is a solution we must show that

$$
\partial_{t} \frac{r w}{t}+\partial_{r} w+\frac{1}{r} w-\frac{\sqrt{t^{2}-r^{2}}}{r t^{2}} \chi_{K}=0
$$

in distribution sense, where $\chi_{K}$ is the characteristic function of the future light cone $K$. Given any test function $\phi$, we must show that

$$
\int_{K}\left(\frac{1}{r} w \phi-\frac{r w}{t} \partial_{t} \phi-w \partial_{r} \phi-\frac{\sqrt{t^{2}-r^{2}}}{r t^{2}} \phi\right) r d r d t=0 .
$$

This amounts to say that, writing

$$
K^{\varepsilon}=\{(t, r): t \geq r+\varepsilon\},
$$

the limit of the same integral over $K^{\varepsilon}$ tends to zero when $\varepsilon \rightarrow 0$. We integrate by parts, the integrals over $K^{\varepsilon}$ cancel since the solution is smooth in $K^{\varepsilon}$ and the terms on the boundary $t=r+\varepsilon$ give (keeping into account the fact that the components $\nu_{t}$ and $\nu_{r}$ of the normal unit vector are opposite)

$$
\int_{\partial K^{\varepsilon}}\left(1-\frac{r}{t}\right) r w \phi d S=\int_{0}^{\infty} \frac{\varepsilon}{r+\varepsilon} r w(r+\varepsilon, r) \phi(r+\varepsilon, r) d r \rightarrow 0 .
$$

It remains now to show that the solutions belong to the stated spaces. For the solution $v$ this follows directly from Lemma 6.1 in Appendix B, and the remark that the $H^{s}$ norms for $s<1$ are controlled by the Besov norm $B_{2, \infty}^{1}$.

Consider now the solution $u$; for $t \leq 0$ it coincides with $v$, hence the same argument applies and we obtain the Besov-valued continuity on $(-\infty, 0]$. We now consider the case $t>0$. We know by Lemma 6.1 in Appendix B that $x_{1} /|x|$ is locally in $B=B_{2, \infty}^{1}$, and this implies that also the function

$$
x_{1}-\frac{x_{1}}{|x|}
$$


is locally in $B$ since $x_{1}$ is smooth. Then also the function

$$
g(x)= \begin{cases}x_{1}-\frac{x_{1}}{|x|} & \text { for }|x| \leq 1 \\ 0 & \text { for }|x| \geq 1\end{cases}
$$

is in $B$ locally, since $g(x)$ is Lipschitz continuous at $|x|=1$. But $g(x)$ is compactly supported, hence $g \in B_{2, \infty}^{1}\left(\mathbb{R}^{2}\right)$ globally. Recalling that the homogeneous norm $\dot{B}_{2, \infty}^{1}\left(\mathbb{R}^{2}\right)$ is invariant for the scaling $x \rightarrow x / t$, we obtain that the function $g(x / t)$ is continuous on $[0,+\infty)$ with values in $B_{2, \infty}^{1}\left(\mathbb{R}^{2}\right)$. This implies immediately that

$$
u_{1}=\frac{x_{1}}{|x|}+g(x / t)
$$

is continuous on $[0,+\infty)$ with values in $B_{2, \infty}^{1}(\Omega)$ for any bounded open $\Omega$. $u_{2}$ is identical. As to $u_{3}$, we only have to remark that

$$
u_{3}(t, x)=\lambda(x / t)
$$

where $\lambda(x)$ is the function defined in Lemma 6.2 in Appendix B, which belongs to $B_{2, \infty}^{1}\left(\mathbb{R}^{2}\right)$, and argue as before.

Continuity with values in $H^{s}$ for $t \neq 0$ follows, since the norm of $B_{2, \infty}^{1}$ is stronger. Continuity also at $t=0$ follows from (3.15) and (3.16) since the scaling properties of $H^{s}$ for $s<1$ imply that

$$
\|g(\cdot / t)\|_{H^{s}\left(\mathbb{R}^{2}\right)} \rightarrow 0 \text { as } t \rightarrow 0 .
$$

The situation does not improve if we consider small solutions. Indeed, we can construct two different weak solutions depending on a parameter $\delta$ which are small and coincide for $t<0$.

The first solution is the self-similar solution obtained using the functions (2.13) with the plus sign; recalling $(2.14),(2.15)$, we have inside the light cones (i.e. for $|x| \leq|t|)$

$$
u_{j}(t, x)=\frac{2 \gamma x_{j}}{\left(1+\gamma^{2}\right) t+\left(1-\gamma^{2}\right) \sqrt{t^{2}-|x|^{2}}}
$$

for $j=1,2$, and

$$
u_{3}(t, x)=\frac{\left(1-\gamma^{2}\right) t+\left(1+\gamma^{2}\right) \sqrt{t^{2}-|x|^{2}}}{\left(1+\gamma^{2}\right) t+\left(1-\gamma^{2}\right) \sqrt{t^{2}-|x|^{2}}}
$$

here $\gamma=\tan \frac{\lambda}{2}$ is a small parameter. On the other hand, outside the cones, i.e. for $|x| \geq|t|$, we have

$$
u_{j}=\frac{x_{j}}{|x|} \delta \operatorname{sn}\left(\arctan \frac{t}{\sqrt{|x|^{2}-t^{2}}} \mid \delta\right) .
$$

for $j=1,2$, and

$$
u_{3}=\operatorname{dn}\left(\arctan \frac{t}{\sqrt{|x|^{2}-t^{2}}} \mid \delta\right) .
$$

Fixed $\delta$ small, we choose $\gamma$ such that

$$
\frac{2 \gamma}{1+\gamma^{2}}=\delta \cdot \operatorname{sn}(\pi / 2 \mid \delta)
$$

to ensure continuity; recall that for $\delta<1$

$$
|\operatorname{sn}(s \mid \delta)| \leq 1, \quad\left|\frac{d}{d s} \operatorname{sn}(s \mid \delta)\right| \leq 1
$$


while

$$
\sqrt{1-\delta^{2}} \leq \operatorname{dn}(s \mid \delta) \leq 1, \quad\left|\frac{d}{d s} \operatorname{dn}(s \mid \delta)\right| \leq \delta .
$$

so the function $u(t, x)$ thus constructed takes its values in a $\delta$-neighborhood of the North pole $(0,0,1)$.

The second solution $v(t, x)$ coincides with $u(t, x)$ for negative $t$ and outside the forward light cone; inside it, i.e., for $t>|x|$, we define it using again (2.13) but we choose now the minus sign; in a more explicit form we obtain (see again (2.14),

$$
v_{j}(t, x)=\frac{2 \gamma x_{j}}{\left(1+\gamma^{2}\right) t-\left(1-\gamma^{2}\right) \sqrt{t^{2}-|x|^{2}}}
$$

for $j=1,2$, and

$$
v_{3}(t, x)=\frac{\left(1-\gamma^{2}\right) t-\left(1+\gamma^{2}\right) \sqrt{t^{2}-|x|^{2}}}{\left(1+\gamma^{2}\right) t-\left(1-\gamma^{2}\right) \sqrt{t^{2}-|x|^{2}}}
$$

(compare with (3.17), (3.18)).

Remark 3.2. Notice that the solution $u(t, x)$ takes its values in a $\delta$-neighborhood of the North pole $(0,0,1)$; on the other hand, the second solution $v(t, x)$ covers the entire sphere, and for $t>0, x=0$ we have $v(t, 0)=(0,0,-1)$, the South pole.

Then we have:

Theorem 3.2. Consider the function $u(t, x)$ defined by (3.17), (3.18) for $|x| \leq|t|$ and by (3.19), (3.20) for $|x|>|t|$. Moreover, consider the function $v(t, x)$ defined as $u(t, x)$ for $t<|x|$ and by (3.22), (3.23) inside the future light cone $t>|x|$. Then both $u(t, x)$ and $v(t, x)$ are weak solutions of the wave map equation (3.1), and have the same regularity properties (3.5). Moreover, for any bounded $\Omega \subseteq \mathbb{R}^{2}$ and $t<0$ fixed we have

$$
\|u(t, \cdot)-N\|_{B_{2, \infty}^{1}(\Omega)} \equiv\|v(t, \cdot)-N\|_{B_{2, \infty}^{1}(\Omega)}=O(\gamma) \text { as } \gamma \rightarrow 0
$$

where $N$ denotes the North pole $N=(0,0,1)$.

Proof. The regularity of $u, v$ is proved as before, and we obtain as above the continuity with values in Besov space for $t>0$ and for $t<0$, with a jump in $t=0(u$ is actually continuous with values in Besov also at $t=0)$. Also the estimate (3.24) follows by a simple argument.

To prove that $u, v$ solve (3.1) we begin as above by applying Lemma 6.3 in the Appendix. The singularity of $u$ and $v$ is concentrated on the light cones $K=\{|t|=$ $|x|\}$. Choosing a ball $\Omega$ near a point of the cone different from the origin, and setting $f=u, h(t, x)=u(|x|, x)$, we see that assumption (6.14) of the Lemma is satisfied, using the Hölder continuity of $u$ near the point; thus the distributional derivative of $u$ on $\left(\mathbb{R} \times \mathbb{R}^{2}\right) \backslash(0,0)$ coincides with the derivative a.e.. Now we can apply again the Lemma choosing $K=$ the origin, and in this case assumption (6.14) follows from the fact that $u$ is bounded near the origin. In conclusion, the first distributional derivative of $u$ on $\mathbb{R} \times \mathbb{R}^{2}$ coincides with its derivative a.e.. The argument for $v$ is identical.

Thus it is sufficient to prove that for any test function $\phi$ the following identity holds:

$$
\int\left[\phi_{t} u_{t} \wedge u-\phi_{r} u_{r} \wedge u+\frac{1}{r} \phi u_{r} \wedge u+\frac{1}{r^{2}} \phi u_{\omega \omega} \wedge u\right] r d r d \omega d t=0
$$


and an analogous one for $v(t, x)$. Now $u$ is a function of the form

$$
u(t, x)=u(t, r \cos \omega, r \sin \omega)=\left(\begin{array}{c}
\cos \omega \sin \psi(r / t) \\
\sin \omega \sin \psi(r / t) \\
\cos \psi(r / t)
\end{array}\right)
$$

and this gives, writing

$$
Z(\omega)=\left(\begin{array}{c}
-\sin \omega \\
\cos \omega \\
0
\end{array}\right)
$$

the identities

$u_{t} \wedge u=Z(\omega) \frac{r}{t^{2}} \psi^{\prime}\left(\frac{r}{t}\right), \quad u_{r} \wedge u=-Z(\omega) \frac{1}{t} \psi^{\prime}\left(\frac{r}{t}\right), \quad u_{\omega \omega} \wedge u=Z(\omega) \sin \psi \cos \psi$.

It is not restrictive to consider test functions of the form $\phi(t, r) \widetilde{\phi}(t, \omega)$. We are thus reduced to prove that

$$
\int\left[\frac{r}{t^{2}} \psi^{\prime}\left(\frac{r}{t}\right) \phi_{t}+\frac{1}{t} \psi^{\prime}\left(\frac{r}{t}\right) \cdot\left(\phi_{r}+\frac{1}{r} \phi\right)+\frac{1}{2 r^{2}} \phi \sin (2 \psi)\right] r d r d t=0 ;
$$

recall that $\psi(s)$ is smooth for $s \neq \pm 1$ and $\psi^{\prime}(s)$ means the derivative a.e.

Introduce now the sets

$$
A_{\varepsilon}=\{|t| \geq|x|+\varepsilon\},
$$

whose boundary is made of the two cones

$$
\partial^{ \pm} A_{\varepsilon}=\{ \pm t=|x|+\varepsilon\},
$$

and

whose boundary is made of the two sets

$$
B_{\varepsilon}=\{|x| \leq|t|+\varepsilon\},
$$

$$
\partial^{+} B_{\varepsilon}=\{t=|x|-\varepsilon, t \geq 0\}, \quad \partial^{-} B_{\varepsilon}=\{-t=|x|-\varepsilon, t \leq 0\} .
$$

Identity (3.25) will follow if we prove that the integral restricted to $A_{\varepsilon} \cup B_{\varepsilon}$ converges to 0 as $\varepsilon \rightarrow 0$. On $A_{\varepsilon} \cup B_{\varepsilon}$ the functions are smooth, hence we can integrate by parts and the integrals on the interior cancel (since $\psi$ solves the self-similar ODE away from the singularity). Only the boundary terms remain, i.e. we must prove that

(3.26) $\int_{\partial+A_{\varepsilon}} \frac{r}{t}\left[\frac{r}{t} \nu_{t}+\nu_{r}\right] \psi^{\prime} \phi d S=C \int_{0}^{\infty} \frac{-\varepsilon r}{(r+\varepsilon)^{2}} \psi^{\prime}\left(\frac{r}{r+\varepsilon}\right) \phi(r+\varepsilon, r) d r \rightarrow 0$,

with a similar relation on $\partial^{-} A_{\varepsilon}$, and

$$
\int_{\partial^{+} B_{\varepsilon}} \frac{r}{t}\left[\frac{r}{t} \nu_{t}+\nu_{r}\right] \psi^{\prime} \phi d S=\int_{0}^{\infty} \frac{t+\varepsilon}{t^{2}} \varepsilon \psi^{\prime}\left(\frac{t+\varepsilon}{t}\right) \phi(t, t+\varepsilon) d t \rightarrow 0
$$

with a similar relation on $\partial^{-} B_{\varepsilon}$. To prove $(3.26),(3.27)$ it is sufficient to recall that $\psi$ satisfies in all cases the condition

$$
\tau^{2}\left(1-\tau^{2}\right) \psi^{\prime}(\tau)^{2}-\sin \psi^{2}=\text { const. }
$$

whence the estimate, valid for any $\tau \neq \pm 1$,

This implies

$$
\left|\psi^{\prime}(\tau)\right| \leq \frac{C}{\tau \sqrt{\left|1-\tau^{2}\right|}}
$$

$$
\left|\psi^{\prime}\left(\frac{r}{r+\varepsilon}\right)\right| \leq C \frac{(r+\varepsilon)^{3 / 2}}{r \sqrt{\varepsilon}}
$$

which gives (3.26), and

$$
\left|\psi^{\prime}\left(\frac{t+\varepsilon}{t}\right)\right| \leq C \frac{t^{2}}{(t+\varepsilon)^{3 / 2} \sqrt{\varepsilon}}
$$


which gives (3.27). The proof for $v$ is identical.

\section{Ill posedness of the Cauchy problem}

This section is devoted to the proof of the following ill-posedness result:

Theorem 4.1. There exists a sequence

$$
h_{k}(t, x) \in L_{\text {loc }}^{1}\left(\mathbb{R} ; L^{2}\left(\mathbb{R}^{2}\right)\right)
$$

such that:

(i) the Cauchy problem

$$
\begin{aligned}
& \phi_{t t}-\Delta \phi+\frac{\sin (2 \phi)}{2|x|^{2}}=h_{k}(t, x), \\
& \phi(0, x)=0, \quad \phi_{t}(0, x)=0
\end{aligned}
$$

has a solution $\phi_{k}(t, x) \in C\left(\mathbb{R} ; H^{1+\varepsilon}\left(\mathbb{R}^{2}\right)\right) \cap C^{1}\left(\mathbb{R} ; H^{\varepsilon}\left(\mathbb{R}^{2}\right)\right)$, where $\varepsilon=\varepsilon(k) \downarrow 0$ as $k \rightarrow \infty$; moreover, both $\phi_{k}(t, x)$ and $h_{k}(t, x)$ are supported inside the forward light cone $t \geq|x|$, and we have the additional smoothness $\phi_{k} \cdot x_{j} /|x| \in C\left(\mathbb{R} ; H^{1+\varepsilon}\left(\mathbb{R}^{2}\right)\right)$;

(ii) for any $T>0$ and all $p, q$ with

$$
\frac{1}{p}+\frac{2}{q}>2
$$

we have, as $k \rightarrow \infty$,

$$
\left\|h_{k}\right\|_{L^{p}\left([-T, T] ; L^{q}\left(\mathbb{R}^{2}\right)\right)} \rightarrow 0
$$

while

$$
\left\|\nabla_{x} \phi_{k}(T, \cdot)\right\|_{L^{2}\left(\mathbb{R}^{2}\right)} \rightarrow \infty, \quad\left\|\partial_{t} \phi_{k}(T, \cdot)\right\|_{L^{2}\left(\mathbb{R}^{2}\right)} \rightarrow \infty .
$$

Remark 4.1. It is not difficult to obtain from this theorem an analogous result for the general wave map equation (1.4). Indeed, set $u=u^{(k)}=\left(u_{1}, u_{2}, u_{3}\right)$ with

$$
u_{1}=\frac{x_{1}}{|x|} \sin \phi_{k}, \quad u_{2}=\frac{x_{2}}{|x|} \sin \phi_{k}, \quad u_{3}=\cos \phi_{k},
$$

with $\phi_{k}$ as in Theorem 4.1. Since $\phi_{k}$ satisfy (4.1), we obtain exactly as in the proof of Theorems 3.1, 3.2 that $u^{(k)}$ is a weak solution of the equation

$$
\square u+\left(\left|u_{t}\right|^{2}-|\nabla u|^{2}\right) u \equiv F_{k}
$$

with

$$
F_{k}=h_{k} \cdot\left(\begin{array}{c}
\frac{x_{1}}{|x|} \cos \phi \\
\frac{x_{2}}{|x|} \cos \phi \\
-\sin \phi
\end{array}\right)
$$

In particular we see that

$$
\left|F_{k}\right|=\left|h_{k}\right|
$$

and this implies

$$
\left\|F_{k}\right\|_{L^{p}\left([-T, T] ; L^{q}\left(\mathbb{R}^{2}\right)\right)} \rightarrow 0
$$

as for $h_{k}$. Notice that when $\phi$ vanishes, $u=(0,0,1)$ (the North pole), while it is immediate to see that $\partial_{t} u(0, x)=0$. Moreover we have easily

$$
\begin{gathered}
\left\|\partial_{t} u(t, \cdot)\right\|_{L^{2}\left(\mathbb{R}^{2}\right)} \equiv\left\|\partial_{t} \phi(t, \cdot)\right\|_{L^{2}\left(\mathbb{R}^{2}\right)}, \\
\left\|\nabla_{x} u(t, \cdot)\right\|_{L^{2}\left(\mathbb{R}^{2}\right)} \equiv\left\|\nabla_{x} \phi(t, \cdot)\right\|_{L^{2}\left(\mathbb{R}^{2}\right)}+\left\||x|^{-1} \phi(t, \cdot)\right\|_{L^{2}\left(\mathbb{R}^{2}\right)}
\end{gathered}
$$

and these relations imply that, for any $T>0$

$$
\left\|\partial_{t} u(T, \cdot)\right\|_{L^{2}\left(\mathbb{R}^{2}\right)} \rightarrow \infty, \quad\left\|\nabla_{x} u(T, \cdot)\right\|_{L^{2}\left(\mathbb{R}^{2}\right)} \rightarrow \infty
$$


In conclusion, we need only check that $u^{(k)} \in C\left(\mathbb{R}, H^{1+\varepsilon}\left(\mathbb{R}^{2}\right)\right)$. We recall that $H^{1+\varepsilon}\left(\mathbb{R}^{2}\right)$ is an algebra. Thus it is standard to see that $\cos \phi-1, \sin \phi$ and in general $g(\phi)$ for $g$ smooth are continuous with values in $H^{1+\varepsilon}\left(\mathbb{R}^{2}\right)$ since $\phi$ is. To prove that $u_{j}=\sin \phi \cdot x_{j} /|x|$ for $j=1,2$ are also smooth we write it as a product

$$
u_{j}=\frac{x_{j}}{|x|} \phi \cdot g(\phi)
$$

with $g(s)=\frac{\sin s}{s}$ analytic; since $g(\phi), x_{j} \phi /|x|$ are in $C H^{1+\varepsilon}$ (see part (i) of the Theorem) the proof is concluded.

Proof. We start from the family of self-similar solution constructed in Section 3 (see $(2.13))$

$$
\psi(\tau, \gamma)=2 \arctan \left(\frac{\gamma \tau}{1+\sqrt{1-\tau^{2}}}\right) .
$$

Inside the future light cone

$$
K=\{(t, x): t>|x|\}
$$

the function $\psi(r / t, \gamma)$ satisfies the equation

$$
\square \psi+\frac{\sin (2 \psi)}{2 r^{2}}=0 .
$$

Notice that if we want to prolong $\psi(r . t, \gamma)$ as a (weak) solution outside $K$ we must resort to the functions given by $(2.13)$ for $\tau>1$; but here we shall not need this second formula.

An alternative form which will be useful in the following is

$$
\psi(\tau, \gamma)=\arcsin (\alpha(\tau, \gamma)), \quad \alpha(\tau, \gamma)=\frac{2 \gamma \tau}{1+\gamma^{2}+\left(1-\gamma^{2}\right) \sqrt{1-\tau^{2}}}
$$

(obtained through the identity $2 \arctan s=\arcsin \left(2 s\left(1+s^{2}\right)^{-1}\right.$ ref $)$ ). Moreover, the derivative of $\psi$ is given by

$$
\frac{\partial}{\partial \tau} \psi(\tau, \gamma)=\frac{2 \gamma}{\sqrt{1-\tau^{2}}} \cdot \frac{1}{1+\gamma^{2}+\left(1-\gamma^{2}\right) \sqrt{1-\tau^{2}}} .
$$

We list here a few consequences of these definitions which will be useful in the following. From (4.9), (4.11) we have immediately

$$
\begin{gathered}
\frac{1}{2} \gamma \tau \leq \psi(\tau, \gamma) \leq 2 \gamma \tau \quad \forall \tau, \gamma \in[0,1] \\
\frac{\gamma}{2 \sqrt{1-\tau^{2}}} \leq \frac{\partial}{\partial \tau} \psi(\tau, \gamma) \leq \frac{2 \gamma}{\sqrt{1-\tau^{2}}} \quad \forall \tau, \gamma \in[0,1]
\end{gathered}
$$

To express the behaviour near $\tau=1$ more precisely we can also write (4.11) as

$$
\frac{\partial}{\partial \tau} \psi(\tau, \gamma)=\frac{2 \gamma}{1+\gamma^{2}} \frac{1}{\sqrt{1-\tau^{2}}}-\frac{2 \gamma^{2}}{1+\gamma^{2}} \frac{1-\gamma^{2}}{1+\gamma^{2}+\left(1-\gamma^{2}\right) \sqrt{1-\tau^{2}}}
$$

and integrating (4.14) from $\tau$ to 1 we obtain

$$
\psi(1, \tau)-\psi(\tau, \gamma)=\frac{2 \gamma}{1+\gamma^{2}}[\arcsin 1-\arcsin \tau-R(\tau, \gamma)]
$$

where the remainder

$$
R(\tau, \gamma)=\int_{\tau}^{1} \frac{\left(1-\gamma^{2}\right) d s}{1+\gamma^{2}+\left(1-\gamma^{2}\right) \sqrt{1-s^{2}}}
$$

satisfies trivially for all $\tau, \gamma \in[0,1]$

$$
0 \leq R(\tau, \gamma) \leq(1-\tau)
$$


Moreover, integrating the elementary identity

$$
\frac{1}{\sqrt{1-s^{2}}}=\frac{1}{\sqrt{2}} \frac{1}{\sqrt{1-s}}+\frac{\sqrt{1-s}}{\sqrt{2} \sqrt{1+s}(\sqrt{2}+\sqrt{1+s})}
$$

from $\tau$ to 1 we get

$$
\arcsin 1-\arcsin \tau=\sqrt{2} \sqrt{1-\tau}+r(\tau), \quad 0 \leq r(\tau) \leq \frac{1}{3}(1-\tau)^{3 / 2} .
$$

In conclusion,

$$
\psi(1, \gamma)-\psi(\tau, \gamma)=\frac{2 \gamma}{1+\gamma^{2}}\left[\sqrt{2} \sqrt{1-\tau}-R_{1}(\tau, \gamma)\right]
$$

where

$$
R_{1}(\tau, \gamma) \equiv R(\tau, \gamma)-r(\tau), \quad 0 \leq R_{1}(\tau, \gamma) \leq(1-\tau) \quad \forall \gamma, \tau \in[0,1]
$$

Now, fix an integer $N$ (which in the end will be taken equal to 2 ; but it is easier to follow the method in the general form), let $\left.\gamma_{1}, \ldots, \gamma_{N} \in\right] 0,1[$ be small positive parameters to be chosen and consider a linear combination of these solutions

$$
\Psi(\tau)=\sum_{j=1}^{N} \mu_{j} \cdot \psi\left(\tau, \gamma_{j}\right)
$$

where the real coefficients $\mu_{j}$ will be precised in the following. Here we impose the condition on $\mu_{j}$

$$
\Psi(1)=0, \quad \text { i.e. } \quad \sum_{j=1}^{N} \mu_{j} \arctan \gamma_{j}=0 .
$$

Notice that this implies using (4.17), (4.18)

$$
\Psi(\tau)=\sum \frac{\mu_{j} \gamma_{j}}{1+\gamma_{j}^{2}}\left[-2 \sqrt{2} \sqrt{1-\tau}+R_{1}\left(\tau, \gamma_{j}\right)\right]
$$

and in particular

$$
|\Psi(\tau)| \leq 6 \sqrt{1-\tau^{2}}\left|\sum \frac{\mu_{j} \gamma_{j}}{1+\gamma_{j}^{2}}\right| .
$$

Then given small positive parameters $A, B \in] 0,1[$ to be chosen we define the approximate solution as

$$
\phi(t, r)= \begin{cases}\rho^{2 A}(1-\tau)^{B} \Psi(\tau) & (t, r) \in K \\ 0 & (t, r) \notin K .\end{cases}
$$

We are using here the hyperbolic coordinates

$$
\rho=\sqrt{t^{2}-r^{2}}, \quad \tau=\frac{r}{t}
$$

inside $K$, thus we can also write

$$
\phi(t, r)=(t-r)^{A+B}(t+r)^{A} t^{-B} \sum_{j=1}^{N} \mu_{j} \psi\left(r / t, \gamma_{j}\right) .
$$

First of all, we check that

$$
\phi \in C\left(\mathbb{R} ; H^{1+\varepsilon}\left(\mathbb{R}^{2}\right)\right) \cap C^{1}\left(\mathbb{R} ; H^{\varepsilon}\left(\mathbb{R}^{2}\right)\right) \quad \forall 0<\varepsilon<A .
$$

It is easy to prove, by arguments similar to those outlined in Lemma 6.2 of the Appendix, that the function

$$
\phi(1, r)=(1-r)^{A+B}(1+r)^{A} \sum \mu_{j} \psi\left(r, \gamma_{j}\right)
$$


(continued as 0 for $r \geq 1$ ) belongs to $H^{1+\varepsilon}\left(\mathbb{R}^{2}\right)$ for any $\varepsilon<A+B$ (and $\varepsilon<1$ ), provided condition (4.20) holds; indeed, (4.20) implies that

$$
\sum \mu_{j} \psi\left(r, \gamma_{j}\right)=\sqrt{1-r^{2}}\left(C+O\left(\sqrt{1-r^{2}}\right)\right)
$$

hence near $r=1$

$$
\phi(1, r)=\left(1-r^{2}\right)^{A+B+1 / 2}\left(C+O\left(\sqrt{1-r^{2}}\right)\right) .
$$

The computations of Lemma 6.2 refer to the special case $A=B=0$. Now, introducing the scaling operators

$$
S_{\lambda} v(x)=v(\lambda x)
$$

we can write for $t>0$

$$
\phi(t, r)=t^{2 A} S_{1 / t} \phi(1, r) .
$$

We recall the well known property $(s \in \mathbb{R})$

$$
\left\|S_{\lambda} v\right\|_{\dot{H}^{s}\left(\mathbb{R}^{n}\right)}=\lambda^{s-n / 2}\|v\|_{\dot{H}^{s}\left(\mathbb{R}^{n}\right)} .
$$

Moreover, we remark that for $v \in \dot{H}^{s}\left(\mathbb{R}^{n}\right)$

$$
\lambda \mapsto S_{\lambda} v \text { is continuous }:\{\lambda \neq 0\} \rightarrow \dot{H}^{s}\left(\mathbb{R}^{n}\right) ;
$$

this is clear for $s=0, v \in C_{0}^{\infty}$ by uniform convergence, follows by density for any $v \in L^{2}$, and this implies the general result through the isomorphism $\dot{H}^{s}\left(\mathbb{R}^{n}\right) \simeq$ $L^{2}\left(|\xi|^{2 s} d \xi\right)$. Thus we see that $t \mapsto \phi(t, r)$, i.e., $t \mapsto t^{2 A} S_{1 / t} \phi(1, r)$, is continuous from $\{t \neq 0\}$ to $H^{1+\varepsilon}$, for any $\varepsilon<A+B, \varepsilon<1$. It only remains to prove continuity as $t \rightarrow 0$; since $\phi \equiv 0$ for $t \leq 0$, this amounts to prove that

$$
\|\phi(t, \cdot)\|_{H^{1+\varepsilon}} \equiv t^{2 A}\left(t \cdot\|\phi(1, \cdot)\|_{L^{2}}+t^{-\varepsilon}\|\phi(1, \cdot)\|_{\dot{H}^{1+\varepsilon}}\right) \rightarrow 0
$$

which is true for $\varepsilon<2 A$ (we have used the properties of the scaling operator $S_{1 / t}$ ). In conclusion, $\phi \in C H^{1+\varepsilon}$ provided $\varepsilon<\min \{1,2 A, A+B\}$.

The proof of the second part of (4.24) is analogous.

We must also check that for $j=1,2$

$$
\frac{x_{j}}{|x|} \phi \in C\left(\mathbb{R} ; H^{1+\varepsilon}\left(\mathbb{R}^{2}\right)\right) ;
$$

by the same rescaling as above, we need only verify that at $t=1$

$$
\frac{x_{j}}{|x|} \phi(1, r) \in H^{1+\varepsilon}\left(\mathbb{R}^{2}\right) .
$$

We already know that $\phi(1, r) \in H^{1+\varepsilon}\left(\mathbb{R}^{2}\right)$, and since $x_{j} /|x|$ is smooth away from 0 , by a cutoff argument we must only prove (4.26) near $x=0$. This is obvious since by the explicit expression of $\phi$ we see that $\phi(1, r)=r \cdot \widetilde{\phi}(r)$ with $\widetilde{\phi} \in C^{\infty}$ near 0 .

We now estimate from below the $H^{1}$ norm of $\phi$. Recall that in the following $0 \leq r \leq 1$ since for $r>1$ we have defined $\phi(1, r)$ as 0 . By the definition

$$
\phi(1, r)=(1-r)^{A+B}(1+r)^{A} \Psi(r),
$$

we have, using (4.11),

$$
\partial_{r} \phi(1, r)=I+I I
$$

where

$$
I=-[2 A r+B(1+r)]\left(1-r^{2}\right)^{A-1}(1-r)^{B} \Psi(r)
$$

and

$$
I I=\left(1-r^{2}\right)^{A-1 / 2}(1-r)^{B} \sum \frac{2 \mu_{j} \gamma_{j}}{1+\gamma_{j}^{2}+\left(1-\gamma_{j}^{2}\right) \sqrt{1-r^{2}}} .
$$


Recalling (4.22), we can bound the first term from above by

$$
|I| \leq 12(A+B)\left(1-r^{2}\right)^{A-1 / 2} \sum\left|\mu_{j}\right| \gamma_{j}
$$

and this gives

$$
\|I\|_{L^{2}} \leq 6(A+B) A^{-1 / 2} \sum\left|\mu_{j}\right| \gamma_{j} .
$$

Using again (as in (4.14)) the identity

$$
\frac{1}{1+\gamma^{2}+\left(1-\gamma^{2}\right) \sqrt{1-r^{2}}}=\frac{1}{1+\gamma^{2}}-\frac{1-\gamma^{2}}{1+\gamma^{2}} \frac{\sqrt{1-r^{2}}}{1+\gamma^{2}+\left(1-\gamma^{2}\right) \sqrt{1-r^{2}}}
$$

we can split the second term as

$$
I I=I I_{1}+I I_{2},
$$

where

$$
I I_{1}=2\left(1-r^{2}\right)^{A-1 / 2}(1-r)^{B} \sum \frac{\mu_{j} \gamma_{j}}{1+\gamma_{j}^{2}}
$$

and

$$
I I_{2}=-2\left(1-r^{2}\right)^{A}(1-r)^{B} \sum \frac{\mu_{j}}{1+\gamma_{j}^{2}+\left(1-\gamma_{j}^{2}\right) \sqrt{1-r^{2}}} \frac{1-\gamma_{j}^{2}}{1+\gamma_{j}^{2}}
$$

an elementary computation gives

$$
\left\|I I_{2}\right\|_{L^{2}} \leq 2 \sum\left|\mu_{j}\right|
$$

provided $\left.A, \gamma_{j} \in\right] 0,1[$, while

$$
\left\|I I_{1}\right\|_{L^{2}} \geq \frac{1}{2}(A+B)^{-1 / 2}\left|\sum \frac{\mu_{j} \gamma_{j}}{1+\gamma_{j}^{2}}\right| .
$$

In conclusion, we have proved that

$$
\|\phi(1, \cdot)\|_{\dot{H}^{1}} \geq \frac{1}{2 \sqrt{A+B}}\left|\sum \frac{\mu_{j} \gamma_{j}}{1+\gamma_{j}^{2}}\right|-6 \frac{A+B}{\sqrt{A}} \sum\left|\mu_{j}\right| \gamma_{j}-2 \sum\left|\mu_{j}\right| .
$$

Finally, to estimate from below the norm of $\phi(t, r)$ it is sufficient to recall that for $t>0$

$$
\|\phi(t, \cdot)\|_{H^{1}} \geq t^{2 A}\|\phi(1, \cdot)\|_{\dot{H}^{1}}
$$

by the scaling properties of $\dot{H}^{1}\left(\mathbb{R}^{2}\right)$. A similar estimate from below holds for $\left\|\partial_{t} \phi(t, \cdot)\right\|_{L^{2}}$.

We now show that the function $\phi$ constructed above is a weak solution of the wave map equation with an appropriate forcing term. First of all, we define a function $g(t, r)$ as follows: $g \equiv 0$ outside the future light cone $K$, while inside $K$ $\left(\rho=\sqrt{t^{2}-r^{2}}, \tau=r / t\right)$

$$
g(t, r)=\left[\square, \rho^{2 A}(1-\tau)^{B}\right] \Psi(r / t)-\rho^{2 A}(1-\tau)^{B} \sum \mu_{j} \frac{\sin \left(2 \psi\left(r / t, \gamma_{j}\right)\right)}{2 r^{2}} .
$$

Notice that inside $K$ we have trivially

$$
\square \phi=\square\left(\rho^{2 A}(1-\tau)^{B} \sum \mu_{j} \psi\left(\tau, \gamma_{j}\right)\right)=g(t, r) ;
$$

our aim is to show that $\square \phi=g$ in distribution sense on $\mathbb{R} \times \mathbb{R}^{2}$.

Since $g \in L_{\text {loc }}^{1}\left(\mathbb{R} \times \mathbb{R}^{2}\right)$, we must show that, for any test function $\chi(t, x)$,

$$
\int(\phi \square \chi-g \chi) d t d x=0 .
$$

Introducing the shifted cone

$$
K_{\varepsilon}=\{(t, x): t \geq|x|+\varepsilon\}
$$


this is equivalent to show that

$$
\int_{K_{\varepsilon}}(\phi \square \chi-g \chi) d t d x \rightarrow 0
$$

as $\varepsilon \rightarrow 0$, since $g$, $\phi$ vanish outside $K$. On $K_{\varepsilon}$ we can integrate by parts and use the identity $\square \phi=g$, hence only boundary terms remain and we are left to show that

$$
\left.\int_{\mathbb{R}^{2}}\left[-\phi \cdot\left(\chi_{t}+\frac{x}{|x|} \cdot \nabla \chi\right)+\chi \cdot\left(\phi_{t}+\phi_{r}\right)\right]\right|_{t=r+\varepsilon} d x \rightarrow 0
$$

The first group of terms is bounded by

$$
C \int_{\Omega}|\phi(r+\varepsilon, r)| r d r
$$

$(\Omega=$ support of $\chi)$ and this quantity tends to 0 since

$$
|\phi(r+\varepsilon, r)|=\varepsilon^{A+B}(2 r+\varepsilon)^{A}(r+\varepsilon)^{-B}|\Psi| \leq C \varepsilon^{A}(2 r+\varepsilon)^{A} .
$$

Thus we only have to show that

$$
\left.\int \chi \cdot\left(\phi_{t}+\phi_{r}\right)\right|_{t=r+\varepsilon} d x \rightarrow 0 .
$$

Recalling that

$$
\phi(t, r)=t^{2 A} \phi(1, r / t)
$$

we have easily

$$
\phi_{r}(t, r)=t^{2 A-1} \phi_{r}(1, r / t)
$$

and

whence

$$
\phi_{t}(t, r)+\phi_{r}(t, r)=2 A t^{-1} \phi(t, r)+t^{-1}(t-r) \phi_{r}(t, r)
$$

$$
\left.\left(\phi_{t}+\phi_{r}\right)\right|_{t=r+\varepsilon}=\frac{2 A}{r+\varepsilon} \phi(r+\varepsilon, r)+\frac{\varepsilon}{r+\varepsilon} \phi_{r}(r+\varepsilon, r) .
$$

The first term is easy to control, since $\phi=\rho^{2 A}(1-\tau)^{B} \Psi$ and $\Psi$ is bounded:

$$
\left|\frac{2 A}{r+\varepsilon} \phi(r+\varepsilon, r)\right|=2 A \varepsilon^{A+B} \frac{(2 r+\varepsilon)^{B}}{(r+\varepsilon)^{1+B}}|\Psi| \leq C A \frac{\varepsilon^{A+B}}{r+\varepsilon}
$$

and of course

$$
\int_{\Omega} \frac{\varepsilon^{A+B}}{r+\varepsilon} r d r \rightarrow 0
$$

The second term is more difficult. Since

$$
\begin{gathered}
\phi_{r}(t, r)=-[2 A r+B(t+r)](t-r)^{A+B-1}(t+r)^{A-1} t^{-B} \Psi(r / t)+ \\
+(t-r)^{A+B}(t+r)^{A} t^{-B-1} \partial_{\tau} \Psi(r / t)
\end{gathered}
$$

we have

$$
\begin{gathered}
\phi_{r}(r+\varepsilon, r)=-[2(A+B) r+B \varepsilon] \frac{\varepsilon^{A+B-1}(2 r+\varepsilon)^{A-1}}{(r+\varepsilon)^{B}} \Psi\left(\frac{r}{r+\varepsilon}\right)+ \\
+\frac{\varepsilon^{A+B}(2 r+\varepsilon)^{A}}{(r+\varepsilon)^{B+1}} \partial_{\tau} \Psi\left(\frac{r}{r+\varepsilon}\right) ;
\end{gathered}
$$

using $|\Psi| \leq C$ and $\left|\partial_{\tau} \Psi(\tau)\right| \leq C\left(1-\tau^{2}\right)^{-1 / 2}$ (see (4.13)) we obtain

$$
\left|\phi_{r}(r+\varepsilon, r)\right| \leq C \varepsilon^{A+B-1}(r+\varepsilon)^{A-B}+C \varepsilon^{A+B-1 / 2}(r+\varepsilon)^{A-B-1 / 2}
$$

which implies

$$
\left|\phi_{r}(r+\varepsilon, r)\right| \leq C \varepsilon^{A-1}(r+\varepsilon)^{A} .
$$


Multiplying by $\varepsilon(r+\varepsilon)^{-1}$ and integrating on the support of $\chi$ we thus obtain

$$
\int_{\Omega} \frac{\varepsilon}{(r+\varepsilon)}\left|\phi_{r}(r+\varepsilon, r)\right| r d r \leq C \int_{\Omega}\left[\varepsilon^{A}(r+\varepsilon)^{A-1}\right] r d r \leq C \varepsilon^{A} \rightarrow 0
$$

and this proves our claim.

We can rewrite the equation $\square \phi=g$ as follows:

$$
\square \phi+\frac{\sin (2 \phi)}{2 r^{2}}=h(t, r)
$$

where $h(t, r)$ is defined as 0 outside $K$ while, inside $K$,

$$
h(t, r)=\left[\square, \rho^{2 A}(1-\tau)^{B}\right] \Psi(r / t)+\frac{\sin (2 \phi)}{2 r^{2}}-\rho^{2 A}(1-\tau)^{B} \sum \mu_{j} \frac{\sin \left(2 \psi\left(r / t, \gamma_{j}\right)\right)}{2 r^{2}} .
$$

We shall now estimate the $L^{p} L^{q}$ norm of $h(t, r)$. To this end we split it as

$$
h=h_{1}+h_{2}
$$

with

$$
h_{1}=\left[\square, \rho^{2 A}(1-\tau)^{B}\right] \Psi(r / t)
$$

and

$$
h_{2}=\frac{\sin (2 \phi)}{2 r^{2}}-\rho^{2 A}(1-\tau)^{B} \sum \mu_{j} \frac{\sin \left(2 \psi\left(r / t, \gamma_{j}\right)\right)}{2 r^{2}} .
$$

Notice that $h_{1}, h_{2}$ are locally integrable functions.

Consider $h_{1}$ first. Using the hyperbolic coordinates $\rho=\sqrt{t^{2}-r^{2}}, \tau=r / t$, inside the future light cone $K$ the commutator can be written

$$
\left[\square, \rho^{2 A}(1-\tau)^{A}\right]=X+c_{1}(\rho, \tau)+c_{2}(\rho, \tau),
$$

where $X$ is the vector field

$$
X=4 A \rho^{2 A-1}(1-\tau)^{B} \frac{\partial}{\partial \rho}+2 B \rho^{2 A-2}(1-\tau)^{B+1}(1+\tau)^{2} \frac{\partial}{\partial \tau}
$$

while $c_{1}, c_{2}$ are the functions

$$
\begin{gathered}
c_{1}(\rho, \tau)=[2 A(2 A+1)-2 B(2 B-1)] \rho^{2 A-2}(1-\tau)^{B}, \\
c_{2}(\rho, \tau)=B \rho^{2 A-2}(1-\tau)^{B+1}\left[3 B+5+(B-1) \tau-\tau^{-1}\right] .
\end{gathered}
$$

Applying $X$ to $\Psi$ which is a function of $\tau$ only, and using formula (4.14) we get

$$
X \Psi=I+I I
$$

where

and

$$
I=2 B \rho^{2 A-2}(1-\tau)^{B+1 / 2}(1+\tau)^{3 / 2} \sum \frac{2 \mu_{j} \gamma_{j}}{1+\gamma_{j}^{2}}
$$

$$
I I=-2 B \rho^{2 A-2}(1-\tau)^{B+1}(1+\tau)^{2} \sum \frac{1-\gamma_{j}^{2}}{1+\gamma_{j}^{2}} \cdot \frac{2 \mu_{j} \gamma_{j}}{1+\gamma_{j}^{2}+\left(1-\gamma_{j}^{2}\right) \sqrt{1-\tau^{2}}} .
$$

Moreover recalling (4.21) we have

$$
c_{1} \Psi=I I I+I V
$$

where

$$
I I I=-4 \sqrt{2}[A(2 A+1)-B(2 B-1)] \rho^{2 A-2}(1-\tau)^{B+1 / 2} \sum \frac{\mu_{j} \gamma_{j}}{1+\gamma_{j}^{2}}
$$

and

$$
|I V|=\left|c_{1}\right| \cdot \sum\left|\mu_{j} \gamma_{j} R_{1}\right| \leq 12(A+B) \rho^{2 A-2}(1-\tau)^{B+1} \sum\left|\mu_{j}\right| \gamma_{j}
$$


Now we have

$$
h_{1}=I+I I+I I I+I V+c_{2} \Psi
$$

the sum $I+I I I$ using (4.17) gives

$$
\begin{aligned}
& I+I I I= \\
& \quad 4 \rho^{2 A-2}(1-\tau)^{B+1 / 2} \sum \frac{\mu_{j} \gamma_{j}}{1+\gamma_{j}^{2}}\left[B(1+\tau)^{3 / 2}-(A(2 A+1)-B(2 B-1)) \sqrt{2}\right] .
\end{aligned}
$$

This term is quite dangerous, i.e. is not in $L^{1} L^{2}$ unless we impose the condition

$$
A=B
$$

which ensures that the quantity in square brackets vanishes at $\tau=1$. With (4.39) we get immediately

$$
|I+I I I| \leq 16 A \rho^{2 A-2}(1-\tau)^{B+3 / 2} \sum\left|\mu_{j}\right| \gamma_{j} .
$$

The remaining terms are quite easy to handle. We have already estimated $I V$, for II we have directly

$$
|I I| \leq 8 B \rho^{2 A-2}(1-\tau)^{B+1} \sum\left|\mu_{j}\right| \gamma_{j}
$$

and for $c_{2} \Psi$ we have, recalling estimate (4.12),

$$
\left|c_{2} \Psi\right| \leq 12 B \rho^{2 A-2}(1-\tau)^{B+1} \sum\left|\mu_{j}\right| \gamma_{j} .
$$

Summing up, we proved that $(A=B)$

$$
\left|h_{1}\right| \leq 64 A \rho^{2 A-2}(1-\tau)^{B+1} \sum\left|\mu_{j}\right| \gamma_{j} .
$$

or equivalently

$$
\left|h_{1}\right| \leq 64 A(t-r)^{A+B}(t+r)^{A-1} t^{-B-1} \sum\left|\mu_{j}\right| \gamma_{j} .
$$

Since for $T>0$, and for all $p, q>0$ such that

$$
\lambda \equiv \frac{1}{p}+\frac{2}{q}-2 \geq 0
$$

we have

we obtain

$$
\left\|(t-r)^{A+B}(t+r)^{A-1} t^{-B-1}\right\|_{L^{p}\left([0, T], L^{q}(t>r)\right)} \leq \frac{T^{\frac{2 A}{p}+\lambda}}{\left[\frac{2 A}{p}+\lambda\right]^{1 / p}}
$$

$$
\left\|h_{1}\right\|_{L^{p}\left([-T, T] ; L^{q}\right)} \leq 64 T^{\frac{2 A}{p}+\lambda} A\left[\frac{2 A}{p}+\lambda\right]^{-1 / p} \sum\left|\mu_{j}\right| \gamma_{j} .
$$

Consider now $h_{2}$, given by

$$
h_{2}=\frac{\sin (2 \phi)}{2 r^{2}}-\rho^{2 A}(1-\tau)^{B} \sum \mu_{j} \frac{\sin \left(2 \psi\left(\tau, \gamma_{j}\right)\right)}{2 r^{2}} .
$$

We can certainly write

$$
\frac{1}{2} \sin (2 s)=s+s^{3} r_{0}(s), \quad\left|r_{0}(s)\right|=\frac{|\cos (\theta)|}{12} \leq 1
$$

where $r_{0}(s)$ is an entire function; thus

$$
\frac{\sin (2 \phi)}{2 r^{2}}=\frac{\rho^{2 A}(1-\tau)^{B}}{r^{2}} \Psi+\frac{\phi^{3}}{r^{2}} r_{0}(\phi) .
$$

Hence recalling that $\Psi=\sum \mu_{j} \psi\left(\tau, \gamma_{j}\right)$ we can split $h_{2}$ as

$$
h_{2}=I+I I
$$


where

$$
I=\frac{2}{r^{2}} \rho^{2 A}(1-\tau)^{B} \sum \mu_{j}\left[2 \arctan \left(\frac{\gamma_{j} \tau}{1+\sqrt{1-\tau^{2}}}\right)-\frac{1}{2} \sin \left(2 \psi\left(\tau, \gamma_{j}\right)\right)\right]
$$

and

$$
I I=\frac{\phi^{3}}{r^{2}} r_{0}(\phi) .
$$

To estimate the quantity $I$ we notice the following identity:

$$
\frac{1}{2} \sin (2 \psi(\tau, \gamma))=\alpha \cdot \sqrt{1-\alpha^{2}}=2 \gamma \tau \frac{1-\gamma^{2}+\left(1+\gamma^{2}\right) \sqrt{1-\tau^{2}}}{\left[1+\gamma^{2}+\left(1-\gamma^{2}\right) \sqrt{1-\tau^{2}}\right]^{2}} ;
$$

this implies, by direct computation,

$$
\begin{aligned}
\frac{\partial}{\partial \tau}\left(2 \arctan \left(\frac{\gamma \tau}{1+\sqrt{1-\tau^{2}}}\right)\right. & \left.-\frac{1}{2} \sin (2 \psi(\tau, \gamma))\right)= \\
& =\frac{16 \gamma^{3} \tau^{3}}{\sqrt{1-\tau^{2}}\left[1+\gamma^{2}+\left(1-\gamma^{2}\right) \sqrt{1-\tau^{2}}\right]^{2}} .
\end{aligned}
$$

Integrating (3.25) from 0 to $\tau$ we obtain easily

$$
0 \leq 2 \arctan \left(\frac{\gamma \tau}{1+\sqrt{1-\tau^{2}}}\right)-\frac{1}{2} \sin (2 \psi(\tau, \gamma)) \leq 32 \gamma^{3} \tau^{3},
$$

and plugging this into (4.43) we obtain

$$
|I| \leq \frac{64}{r^{2}} \rho^{2 A}(1-\tau)^{B} \tau^{3} \sum\left|\mu_{j}\right| \gamma_{j}^{3} .
$$

As to the remainder, we can use the inequality $|\psi(\tau, \gamma)| \leq 2 \gamma \tau$ to obtain

$$
|\phi|^{3} \leq 8 \rho^{6 A}(1-\tau)^{3 B} \tau^{3}\left|\sum \mu_{j} \gamma_{j}\right|^{3} \leq 8 N^{3} \rho^{6 A}(1-\tau)^{3 B} \tau^{3} \sum\left|\mu_{j}\right|^{3} \gamma_{j}^{3}
$$

and this implies

In conclusion

$$
|I I| \leq 8 N^{3} \frac{1}{r^{2}} \rho^{2 A}(1-\tau)^{B} \tau^{3} \sum\left|\mu_{j}\right|^{3} \gamma_{j}^{3} .
$$

$$
\left|h_{2}\right|=|I+I I| \leq 64 N^{3} \frac{1}{r^{2}} \rho^{2 A}(1-\tau)^{B} \tau^{3} \sum\left(\left|\mu_{j}\right|+\left|\mu_{j}\right|^{3}\right) \gamma_{j}^{3} ;
$$

since

and, for $T>0$,

$$
\frac{1}{r^{2}} \rho^{2 A}(1-\tau)^{B} \tau^{3}=(t-r)^{A+B}(t+r)^{A} t^{-B-3} r
$$

$$
\left\|(t-r)^{A+B}(t+r)^{A} t^{-B-3} r\right\|_{L^{p}\left([0, T], L^{q}(t>r)\right)} \leq 2 \frac{T^{\frac{2 A}{p}+\lambda}}{\left[\frac{2 A}{p}+\lambda\right]}
$$

where $\lambda \geq 0$ is given by (4.41), we have proved that

$$
\left\|h_{2}\right\|_{L^{p}\left([-T, T] ; L^{q}\right)} \leq 128 N^{3} T^{\frac{2 A}{p}+\lambda}\left[\frac{2 A}{p}+\lambda\right]^{-1 / p} \sum\left(\left|\mu_{j}\right|+\left|\mu_{j}\right|^{3}\right) \gamma_{j}{ }^{3} .
$$

In conclusion, by (4.42) and (4.47) we find (4.48)

$$
\|h\|_{L^{p}\left([-T, T] ; L^{q}\right)} \leq 128 N^{3} T^{\frac{2 A}{p}+\lambda}\left[\frac{2 A}{p}+\lambda\right]^{-1 / p} \sum\left|\mu_{j}\right| \gamma_{j}\left[A+\gamma_{j}^{2}\left(1+\left|\mu_{j}\right|^{2}\right)\right] .
$$

We are now ready to choose the parameters. Given a small parameter $a>0$ we shall express all the other quantities in terms of it. First of all we take

$$
\gamma_{j}=\tan (a \cdot j), \quad j=1,2, \ldots
$$


so that the condition (4.20) on $\mu_{j}$ becomes

$$
\sum_{j=1}^{N} \mu_{j} \cdot j=0
$$

clearly it is sufficient to take

$$
N=2, \quad \mu_{1}=2, \quad \mu_{2}=-1 .
$$

Notice that

$$
\frac{2 \gamma_{j}}{1+\gamma_{j}^{2}}=\sin (a j / 2)
$$

Then estimate (4.29) gives for $t>0$

$$
\|\phi(T, \cdot)\|_{H^{1}} \geq T^{A} A^{-1 / 2}(2 \sin (a / 2)-\sin (a)) \geq C T^{A} A^{-1 / 2} a^{3} .
$$

Since we want this quantity to be unbounded as $a \rightarrow 0$, we must choose

$$
A=a^{\delta+6}
$$

for some $\delta>0$. Then estimate (4.48) gives

$$
\|h\|_{L^{p} L^{q}} \leq C T^{\frac{2 A}{p}+\lambda}\left[\frac{2 a^{6+\delta}}{p}+\lambda\right]^{-1 / p} a^{3} .
$$

This quantity tends to 0 as $a \rightarrow 0$ when $\lambda>0$, i.e., when condition (4.3) holds. This concludes the proof of our theorem.

\section{Appendix A: The self-similar OdE}

This section is devoted to a complete study of the equation

$$
\tau^{2}\left(\tau^{2}-1\right) \psi^{\prime \prime}+\tau\left(2 \tau^{2}-1\right) \psi^{\prime}+k^{2} \sin \psi \cos \psi=0
$$

which governs the profile of self-similar solutions $\phi(t, r)=\psi(r / t)$ to equation (2.5). Here $k \geq 1$ is any integer.

We can express all the solutions to (5.1) in an explicit form using Jacobi elliptic functions. These functions are usually introduced as doubly periodic meromorphic functions with suitable additional properties; but the standard definition is only given for restricted values the parameters, hence from our standpoint it is both too general and too restrictive. For convenience of the reader, we construct them from scratch in a very short but complete way.

Remark 5.1. JACOBI ElliptiC FUnCtions ON $\mathbb{R}$. Consider the system of ODEs for the functions $f, g, h: \mathbb{R} \rightarrow \mathbb{R}$

$$
\begin{aligned}
& f^{\prime}=g h \\
& g^{\prime}=-h f \\
& h^{\prime}=-m^{2} f g
\end{aligned}
$$

where $m$ is a fixed real number, subject to the initial conditions

$$
f(0)=0, \quad g(0)=1, \quad h(0)=1 .
$$

It is clear that the system admits a unique $C^{1}$ solution $(f, g, h)$ which is in fact real analytic and can be prolonged on the whole $\mathbb{R}$ using the first integrals of the system

$$
f^{2}+g^{2}=1, \quad m^{2} f^{2}+h^{2}=1, \quad m^{2} g^{2}-h^{2}=1
$$

which follow at once from the equations. The standard notation for these functions is

$$
f=\operatorname{sn}(t \mid m), \quad g=\operatorname{cn}(t \mid m), \quad h=\operatorname{dn}(t \mid m) .
$$


Notice that in the literature the parameter $m$ is usually restricted to the range $[0,1[$, which is not sufficient for our purposes. It is clear by the definition that

$$
\operatorname{sn}(t \mid m)=\operatorname{sn}(t \mid-m), \quad \operatorname{cn}(t \mid m)=\operatorname{cn}(t \mid-m), \quad \operatorname{dn}(t \mid m)=\operatorname{dn}(t \mid-m) .
$$

Squaring the equations and using the conservation laws (5.6), we obtain immediately

$$
\begin{gathered}
{f^{\prime}}^{2}=\left(1-f^{2}\right)\left(1-m^{2} f^{2}\right), \\
g^{\prime 2}=\left(1-g^{2}\right)\left(1-m^{2}+m^{2} g^{2}\right), \\
{h^{\prime}}^{2}=-\left(1-h^{2}\right)\left(1-m^{2}-h^{2}\right) .
\end{gathered}
$$

From (5.7)-(5.9) it is immediate to obtain several properties of the elliptic functions.

Consider first the case $|m|<1$. Then $\mathrm{sn}(t \mid m)$ and $\mathrm{cn}(t \mid m)$ are periodic with period $4 K(m)$, where

$$
K(m)=\int_{0}^{\pi / 2} \frac{d s}{\sqrt{1-m^{2} \sin ^{2} s}}
$$

is called the complete integral of the first species. The couple $(\operatorname{sn}(t \mid m), \mathrm{cn}(t \mid m))$ has a behaviour similar to the couple ( $\sin , \cos )$, i.e., they oscillate between \pm 1 , and indeed we have in the special case $m=0$

$$
\operatorname{sn}(t \mid 0)=\sin t, \quad \operatorname{cn}(t \mid 0)=\cos t .
$$

Moreover the zeroes of $\mathrm{sn}(t \mid m)$ are $t=2 j K(m)$ and those of $\mathrm{cn}(t \mid m)$ are $(2 j+$ $1) K(m), j \in \mathbb{Z}$. On the other hand, the function $\operatorname{dn}(t \mid m)$ has period $2 K(m)$ and oscillates between the values 1 and $\sqrt{1-m^{2}}$, thus it is strictly positive (and is identically 1 when $m=0$ ). A fundamental property connecting elliptic functions with elliptic integrals is the following: for fixed $|m|<1$,

$$
\text { if } \int_{0}^{\alpha} \frac{d s}{\sqrt{1-m^{2} \sin ^{s} s}}=\beta \text { then } \sin \alpha=\operatorname{sn}(\beta \mid m), \cos \alpha=\operatorname{cn}(\beta \mid m) .
$$

This follows from (5.7), (5.8) through the change of variables $f \rightarrow \sin f, g \rightarrow \cos g$.

When $|m|>1$ the behaviour changes. The function sn $(t \mid m)$ oscillates between $\pm 1 / m$, and indeed we have the formula

$$
\operatorname{sn}(t \mid m)=\frac{1}{m} \operatorname{sn}\left(m t \mid m^{-1}\right)
$$

from which we see that the period is $4 K\left(m^{-1}\right) m^{-1}$ and the zeroes $2 j K\left(m^{-1}\right) m^{-1}$. The functions $\mathrm{cn}(t \mid m)$ and $\operatorname{dn}(t \mid m)$ exchange with each other according to the formulas

$$
\operatorname{cn}(t \mid m)=\operatorname{dn}\left(m t \mid m^{-1}\right), \quad \operatorname{dn}(t \mid m)=\operatorname{cn}\left(m t \mid m^{-1}\right)
$$

so that, for $|m|>1, \mathrm{cn}(t \mid m)$ is strictly positive, oscillates between 1 and $\sqrt{1-m^{-2}}$ and has period $2 K\left(m^{-1}\right) m^{-1}$, while $\operatorname{dn}(t \mid m)$ oscillates between \pm 1 and has period $4 K\left(m^{-1}\right) m^{-1}$.

When $|m|=1$ we have simply

$$
\operatorname{sn}(t \mid 1)=\tanh t=\frac{e^{t}-e^{-t}}{e^{t}+e^{-t}}, \quad \operatorname{cn}(t \mid 1)=\operatorname{dn}(t \mid 1)=\frac{1}{\cosh t}=\frac{2}{e^{t}+e^{-t}} .
$$

We finally introduce the fourth Jacobi function called the amplitude and connected to the above through the relations

$$
\operatorname{sn}(t \mid m)=\sin (\operatorname{am}(t \mid m)), \quad \operatorname{cn}(t \mid m)=\cos (\operatorname{am}(t \mid m)) .
$$


When $|m|<1$ we can compute for $|t|<K(m)$ (i.e., between the first zeroes of cn)

$$
\operatorname{am}(t \mid m)=\arctan \frac{\operatorname{sn}(t \mid m)}{\operatorname{cn}(t \mid m)} \text {. }
$$

Actually am $(t \mid m)$ extends as an analytic function for all $t \in \mathbb{R}$; indeed, by (5.11) we have immediately

$$
\text { if } \int_{0}^{\alpha} \frac{d s}{\sqrt{1-m^{2} \sin ^{s} s}}=\beta \quad \text { then } \alpha=\operatorname{am}(\beta \mid m) \quad(|m|<1)
$$

which means that $\mathrm{am}(t \mid m)$ is the inverse function of the integral to the right, regarded as a function of $\alpha$. This equivalent definition of am $(t \mid m)$ is meaningful for any $t$, provided $|m|<1$.

When $|m|>1$ we can use for all $t$ definition (5.16), since $c n(t \mid m)$ is strictly positive then; thus am $(t \mid m)$ is $4 K\left(m^{-1}\right) / m^{-1}$ periodic and oscillates between the values $\pm \arctan \left[\left(m^{2}-1\right)^{-1 / 2}\right]$. Notice that also in this case am $(t \mid m)$ inverts the elliptic integral as in (5.17), but only on a finite interval:

$$
\text { if } \int_{0}^{\alpha} \frac{d s}{\sqrt{1-m^{2} \sin ^{s} s}}=\beta \quad \text { then } \alpha=\operatorname{am}(\beta \mid m) \quad\left(|m|>1,|\alpha|<\arcsin \left(|m|^{-1}\right)\right)
$$

The zeroes of am $(t \mid m)$ are the same of $\operatorname{sn}(t \mid m)$, that is to say $2 j K\left(m^{-1}\right) m^{-1}$. When $|m|=1$ we have

$$
\operatorname{am}(t \mid 1)=2 \arctan \left(e^{t}\right)-\frac{\pi}{2}=2 \arctan \frac{e^{t}-1}{e^{t}+1} .
$$

We are now ready to prove the

Proposition 5.1. Consider the equation

$$
\tau^{2}\left(\tau^{2}-1\right) \psi^{\prime \prime}+\tau\left(2 \tau^{2}-1\right) \psi^{\prime}+k^{2} \sin \psi \cos \psi=0 .
$$

(i) If $\psi$ is a solution, also $j \pi \pm \psi$ are solutions, $j \in \mathbb{Z}$. (Thus it is sufficient to study the solutions in the range $[0, \pi / 2]$ ). The only constant solutions are given by $\psi(\tau)=j \pi / 2$.

(ii) If $\psi(\tau)$ is a $C^{1}$ solution near a point $\left.\tau_{0} \in\right] 0,1[$, then $\psi$ can be extended to an analytic function on the whole interval $] 0,1[$. Moreover, the limits

$$
\lambda=\lim _{\tau \uparrow 1} \psi(\tau), \quad \mu=\lim _{\tau \uparrow 1} \psi^{\prime}(\tau) \sqrt{1-\tau^{2}}
$$

exist and characterize uniquely the solution $\psi$. Indeed, for $\lambda \in] 0, \pi / 2[$ and any $\mu$, or for $\lambda=0, \pi / 2$ and $\mu \neq 0$ (the excluded cases correspond to the constant solutions), we can represent $\psi(\tau)$ as follows:

$$
\psi(\tau)=\frac{\pi}{2}-\operatorname{am}\left(\left(1+q_{0}\right)^{1 / 2}\left[q_{1}+\operatorname{sgn} \mu \cdot k \cdot \operatorname{arctanh} \sqrt{1-\tau^{2}}\right] \mid\left(1+q_{0}\right)^{-1 / 2}\right)
$$

where

$$
q_{0}=\frac{\mu^{2}}{k^{2}}-\sin ^{2} \lambda, \quad q_{1}=\int_{\lambda}^{\pi / 2} \frac{d s}{\sqrt{q_{0}+\sin ^{2} s}}
$$

and $\operatorname{sgn} \mu$ must be replaced by -1 when $\mu=0$.

(iii) If $\psi(\tau)$ is a $C^{1}$ solution near a point $\tau_{0}>1$, then $\psi$ can be extended to an analytic function on the whole interval $] 1, \infty[$. Moreover, the limits

$$
\lambda=\lim _{\tau \downarrow 1} \psi(\tau), \quad \mu=\lim _{\tau \downarrow 1} \psi^{\prime}(\tau) \sqrt{\tau^{2}-1}
$$


exist and characterize uniquely the solution $\psi$. Indeed, for $\lambda \in] 0, \pi / 2[$ and any $\mu$, or for $\lambda=0, \pi / 2$ and $\mu \neq 0$ (the excluded cases correspond to the constant solutions), we can represent $\psi(\tau)$ as follows:

$$
\psi(\tau)=\operatorname{am}\left(q_{0}^{1 / 2}\left[q_{1}+\operatorname{sgn} \mu \cdot k \cdot \arctan \sqrt{\tau^{2}-1}\right] \mid q_{0}^{-1 / 2}\right)
$$

where

$$
q_{0}=\frac{\mu^{2}}{k^{2}}+\sin ^{2} \lambda, \quad q_{1}=\int_{0}^{\lambda} \frac{d s}{\sqrt{q_{0}-\sin ^{2} s}}
$$

and $\operatorname{sgn} \mu$ must be replaced by -1 when $\mu=0$.

Remark 5.2. Before sketching the proof, we single out a few solutions with special properties that are used in the paper.

(a) For $\tau \in] 0,1[$, most of the solutions given by (5.21) have a nasty behaviour near 0 , indeed arctanh is unbounded near 1 and am $(t \mid m)$ either has a linear growth $(|m|<1)$ or oscillates $(|m|>1)$. The only good solutions are obtained when $q_{0}=0$, i.e., with the choice

$$
\lambda \in] 0, \pi / 2], \quad \mu= \pm k \sin \lambda \quad \Rightarrow \quad q_{0}=0, \quad q_{1}=\int_{\lambda}^{\pi / 2} \frac{d s}{\sin s}=-\log \tan \frac{\lambda}{2}
$$

recalling (5.19) and the identities

$$
\exp \left(\operatorname{arctanh} \sqrt{1-\tau^{2}}\right)=\frac{1+\sqrt{1-\tau^{2}}}{\tau}, \quad \frac{\pi}{2}-\arctan x=\arctan \frac{1}{x}
$$

we obtain

$$
\psi(\tau)=2 \arctan \left[\tan \frac{\lambda}{2} \cdot\left(\frac{\tau}{1 \pm \sqrt{1-\tau^{2}}}\right)^{k}\right]
$$

where $\pm=\operatorname{sgn} \mu$. It is useful to compute also

$$
\sin \psi(\tau)=\frac{2 \gamma \tau^{k}}{\left(1 \pm \sqrt{1-\tau^{2}}\right)^{k}+\gamma^{2}\left(1 \mp \sqrt{1-\tau^{2}}\right)^{k}}, \quad \gamma=\tan \frac{\lambda}{2},
$$

and

$$
\cos \psi(\tau)=\frac{\left(1 \pm \sqrt{1-\tau^{2}}\right)^{k}-\gamma^{2}\left(1 \mp \sqrt{1-\tau^{2}}\right)^{k}}{\left(1 \pm \sqrt{1-\tau^{2}}\right)^{k}+\gamma^{2}\left(1 \mp \sqrt{1-\tau^{2}}\right)^{k}}, \quad \gamma=\tan \frac{\lambda}{2},
$$

where $\pm=\operatorname{sgn} \mu($ and $\mp=-\operatorname{sgn} \mu)$.

(b) The solutions for $\tau>1$ have a nice behaviour, and in particular they are monotone; we shall be interested in small solutions with the property $\psi \rightarrow 0$ as $\tau \rightarrow \infty$. Actually we have:

for any fixed $k \geq 1$ and small $\delta>0$ we can find (unique) $\lambda, \mu$ such that $q_{0}=\delta^{2}$ and the solution given by (5.23) tends to 0 as $\tau \rightarrow+\infty$. The solution is monotone decreasing for odd $k$ and monotone increasing for even $k$. In particular, for $k=1$ we can choose $q_{1}=\pi / 2$ and the solution is given by

$$
\psi(\tau)=\operatorname{am}\left(\delta \arctan \frac{1}{\sqrt{\tau^{2}-1}} \mid \frac{1}{\delta}\right),
$$

so that, recalling formulas (5.13),

$$
\sin \psi(\tau)=\delta \cdot \operatorname{sn}\left(\arctan \frac{1}{\sqrt{\tau^{2}-1}} \mid \delta\right),
$$




$$
\cos \psi(\tau)=\operatorname{dn}\left(\arctan \frac{1}{\sqrt{\tau^{2}-1}} \mid \delta\right),
$$

Indeed, since $q_{0}<1$, the amplitude function in (5.23) has a periodic behaviour, with zeroes in the points $2 j K\left(q_{0}^{1 / 2}\right) q_{0}^{1 / 2}$. Recall that

$$
K\left(q_{0}^{1 / 2}\right)=\int_{0}^{\pi / 2} \frac{d s}{\sqrt{1-q_{0} \sin ^{2} s}}=\int_{0}^{\arcsin \sqrt{q_{0}}} \frac{d s}{\sqrt{q_{0}-\sin ^{2} s}},
$$

while

$$
q_{1}=\int_{0}^{\lambda} \frac{d s}{\sqrt{q_{0}-\sin ^{2} s}} .
$$

Notice that $q_{1} \leq K\left(q_{0}^{1 / 2}\right)$ for small $\lambda, \mu$ since

$$
\lambda \leq \arcsin \sqrt{q_{0}}=\arcsin \sqrt{\sin ^{2} \lambda+\mu^{2} / k^{2}} ;
$$

more precisely, if we keep $q_{0}$ fixed and change the value of $\lambda$, $\mu$, we see that $q_{1}$ takes all the values from 0 (when $\lambda=0$ ) to $K\left(q_{0}^{1 / 2}\right.$ ) (when $\mu=0$ ). Moreover, $K(m)$ is a strictly increasing function for $0<m<1$, with $K(m) \downarrow \pi / 2$ as $m \downarrow 0$ and $K(m) \uparrow \infty$ as $m \uparrow 1$. Now the solution (5.23) tends to 0 as $\tau \rightarrow \infty$ provided the $\operatorname{argument} s$ of am $\left(s \mid q_{0}^{-1 / 2}\right)$ approaches one of the zeroes, i.e., provided we can find $j \in \mathbb{Z}$ such that

$$
q_{1}+\operatorname{sgn} \mu \cdot k \frac{\pi}{2}=2 j K\left(q_{0}^{1 / 2}\right) .
$$

If $k>0$ is odd, we can write $k=-2 j+1$ for a negative integer $j$ and choosing $\mu<0$ condition (5.32) becomes

$$
q_{1}=\frac{\pi}{2}+|2 j|\left(\frac{\pi}{2}-K\left(q_{0}^{1 / 2}\right)\right) .
$$

The right hand side is slightly less than $\pi / 2$ for small $q_{0}$, and keeping the value of $q_{0}$ fixed we can find $\lambda, \mu$ such that the condition is satisfied (since $q_{1}$ ranges from 0 to $K\left(q_{0}^{1 / 2}\right)>\pi / 2$ ). Thus our claim is proved for odd $k$. A similar argument holds for even $k$ (we choose now $2 j=k$ and $\mu>0$ ).

Proof. The claims of part (i) are self-evident. We now prove (ii). It is clear that $\psi$ defined near $\left.\tau_{0} \in\right] 0,1\left[\right.$ is analytic; if we multiply the equation by $\psi^{\prime}$ we obtain the identity

i.e.,

$$
\left[\tau^{2}\left(1-\tau^{2}\right) \psi^{\prime}(\tau)^{2}-k^{2} \sin ^{2} \psi\right]^{\prime}=0,
$$

$$
\tau^{2}\left(1-\tau^{2}\right) \psi^{\prime}(\tau)^{2}-k^{2} \sin ^{2} \psi=\text { const } .
$$

A first consequence is that we can extend the solution of (5.20) $\psi$ on the whole interval $] 0,1\left[\right.$ since $\psi^{\prime}$ must be bounded on any compact subinterval. Moreover, setting $g\left(\sqrt{1-\tau^{2}}\right)=\psi(\tau)$, we see that the function $g(s)$ satisfies the differential equation

$$
\tau^{4} g^{\prime}\left(\sqrt{1-\tau^{2}}\right)^{2}-k^{2} \sin ^{2} g\left(\sqrt{1-\tau^{2}}\right)=\text { const } .
$$

i.e.,

$$
\left(1-s^{2}\right)^{2} g^{\prime}(s)^{2}-k^{2} \sin ^{2} g(s)=\text { const. }
$$

Thus we see that $g(s)$ is an analytic function near $s=0$, and this implies that both $\psi(\tau)=g\left(\sqrt{1-\tau^{2}}\right)$ and $\sqrt{1-\tau^{2}} \psi^{\prime}(\tau)=-\tau g^{\prime}\left(\sqrt{1-\tau^{2}}\right)$ have a limit as $\tau \uparrow 1$, as claimed. Defining $\lambda, \mu, q_{0}, q_{1}$ as in the statement, we can write

$$
\tau^{2}\left(1-\tau^{2}\right) \psi^{\prime}(\tau)^{2}-k^{2} \sin ^{2} \psi=q_{0} k^{2}
$$


or equivalently

$$
\frac{\psi^{\prime}(\tau)^{2}}{q_{0}+\sin ^{2} \psi}=\frac{k^{2}}{\tau^{2}\left(1-\tau^{2}\right)}
$$

and hence

$$
\frac{\psi^{\prime}(\tau)}{\left(q_{0}+\sin ^{2} \psi\right)^{1 / 2}}= \pm \frac{k}{\tau \sqrt{1-\tau^{2}}}
$$

where $\pm=\operatorname{sgn} \mu$. To solve this we set $\chi=-\psi+\pi / 2$ and obtain

$$
\frac{\chi^{\prime}(\tau)}{\left(q_{0}+1-\sin ^{2} \chi\right)^{1 / 2}}=\mp \frac{k}{\tau \sqrt{1-\tau^{2}}} .
$$

We can now integrate between $\tau$ and 1 ; for $\tau$ close enough to 1 , recalling (5.17), we obtain immediately that (5.21) holds in a left neighborhood of 1 and hence on ]0, 1 [ by analyticity.

The proof of part (iii) is analogous.

\section{Appendix B: Some technical Lemmas}

We recall the standard definitions

$$
\|u\|_{B_{2, \infty}^{1}\left(\mathbb{R}^{2}\right)}=\|u\|_{L^{2}}+\sup _{j \in \mathbb{Z}} 2^{j}\|\widehat{u}\|_{L^{2}\left(2^{j-1} \leq|\xi| \leq 2^{j+1}\right)},
$$

and, for any open set $\Omega$,

$$
\|u\|_{B_{2, \infty}^{1}(\Omega)}=\inf \left\{\left\|u_{1}\right\|_{B_{2, \infty}^{1}\left(\mathbb{R}^{2}\right)}: u_{1} \in B_{2, \infty}^{1}\left(\mathbb{R}^{2}\right),\left.u_{1}\right|_{\Omega}=u\right\}
$$

Of course $B_{2, \infty}^{1}(\Omega)$ can be defined as the space of restrictions to $\Omega$ of functions in $\|u\|_{B_{2, \infty}^{1}\left(\mathbb{R}^{2}\right)}$, which in turn are the temperate distributions for which the above norm is defined and finite. For details see [19], §4.2.1.

Lemma 6.1. The function

$$
\theta(x)=\frac{x}{|x|}
$$

belongs locally to the Besov space $B_{2, \infty}^{1}$, i.e., it belongs to $B_{2, \infty}^{1}(\Omega)$ for any bounded open set $\Omega \subseteq \mathbb{R}^{2}$.

Proof. By the definition of $B_{2, \infty}^{1}(\Omega)$, it is sufficient to show that $\psi(x) \theta(x)$ is in $B_{2, \infty}^{1}\left(\mathbb{R}^{2}\right)$ for any cutoff function $\psi \in C_{c}^{\infty}\left(\mathbb{R}^{n}\right)$. First of all we recall that the Fourier transform of the $L^{\infty}\left(\mathbb{R}^{2}\right)$ function $\theta(x)$ can be expressed as

$$
\widehat{\theta}(\xi)=P . V \cdot \frac{\xi}{|\xi|^{3}}=\lim _{\varepsilon \downarrow 0} \chi_{\varepsilon} \frac{\xi}{|\xi|^{3}}, \quad \chi_{\varepsilon}(\xi)=1 \text { for }|\xi| \geq \varepsilon, 0 \text { elsewhere }
$$

where P.V. (meaning principal value) is exactly defined as the limit in distribution sense at the right hand side (see e.g. [15], p.164 ff.). Thus we are led to estimate the quantities

$$
\left\|\int_{|\xi| \geq \varepsilon} \widehat{\psi}(\xi-\eta) \frac{\eta}{|\eta|^{3}} d \eta\right\|_{L^{2}\left(2^{j-1} \leq|\xi| \leq 2^{j+1}\right)}
$$

uniformly with respect to $\varepsilon$. We split the integral as follows

$$
\int_{|\eta| \geq \varepsilon} \widehat{\psi}(\xi-\eta) \frac{\eta}{|\eta|^{3}} d \eta=\int_{1 \geq|\eta| \geq \varepsilon}+\int_{|\eta| \geq 1}=I_{\varepsilon}(\xi)+I I(\xi) .
$$

The first part can be handled by the standard trick

$$
I_{\varepsilon}=\int_{1 \geq|\eta| \geq \varepsilon} \widehat{\psi}(\xi-\eta) \frac{\eta}{|\eta|^{3}} d \eta=\int_{1 \geq|\eta| \geq \varepsilon}[\widehat{\psi}(\xi-\eta)-\widehat{\psi}(\xi)] \frac{\eta}{|\eta|^{3}} d \eta
$$


since $\eta$ has average 0 on the sphere. We recall now that $\widehat{\psi}$ is rapidly decreasing and hence satisfies

$$
\left|\nabla_{\eta} \widehat{\psi}(\eta)\right|+|\widehat{\psi}(\eta)| \leq C_{N}\langle\eta\rangle^{-N}, \quad\langle\eta\rangle^{2}=1+|\eta|^{2}
$$

for any $N$; thus by Taylor's formula, if $|\eta| \leq 1$,

$$
|\widehat{\psi}(\xi-\eta)-\widehat{\psi}(\xi)| \leq \sup _{0 \leq \theta \leq 1}\left|\nabla_{\eta} \widehat{\psi}(\xi-\theta \eta)\right| \cdot|\eta| \leq C_{N}\langle\xi\rangle^{-N}|\eta|
$$

where $C_{N}$ is independent of $\varepsilon$. This gives

$$
\left|I_{\varepsilon}(\xi)\right| \leq C_{N}\langle\xi\rangle^{-N} \int_{|\eta| \leq 1}|\eta|^{-1} d \eta \leq C_{N}\langle\xi\rangle^{-N}
$$

whence, choosing $N=3$,

$$
\left\|I_{\varepsilon}\right\|_{L^{2}\left(2^{j-1} \leq|\xi| \leq 2^{j+1}\right)} \leq C_{0} 2^{-j}
$$

with $C_{0}$ independent of $\varepsilon$.

We now estimate $I I(\xi)$, which corresponds to the integration on $|\eta| \geq 1$. When $|\xi| \leq 1$ it is sufficient to remark that for any $N$

$$
|\widehat{\psi}(\xi-\eta)| \leq C\langle\eta\rangle^{-N}
$$

and this gives immediately

$$
\|I I\|_{L^{2}(|\xi| \leq 1)} \leq C
$$

It remains to consider the $L^{2}$ norm of $I I(\xi)$ when $|\xi| \sim 2^{j}, j \geq 0$. We can split $I I$ as

$$
I I(\xi)=\int_{|\eta| \leq|\xi| / 2} \widehat{\psi}(\xi-\eta) \frac{\eta}{|\eta|^{3}} d \eta+\int_{|\eta| \geq|\xi| / 2} \widehat{\psi}(\xi-\eta) \frac{\eta}{|\eta|^{3}} d \eta=I I_{1}+I I_{2} .
$$

When $|\eta| \leq|\xi| / 2$ we have

$$
|\widehat{\psi}(\xi-\eta)| \leq C\langle\xi-\eta\rangle^{-N} \leq C\langle\xi\rangle^{-N}
$$

which implies

$$
\left\|I I_{1}\right\|_{L^{2}\left(2^{j-1} \leq|\xi| \leq 2^{j+1}\right)} \leq C 2^{-j}
$$

on the other hand, when $|\eta| \geq|\xi| / 2$, we have directly

$$
\left|I I_{2}(\xi)\right| \leq|\xi|^{-2} \int|\widehat{\psi}(\xi-\eta)| d \eta \leq C|\xi|^{-2}
$$

and this gives

$$
\left\|I I_{2}\right\|_{L^{2}\left(2^{j-1} \leq|\xi| \leq 2^{j+1}\right)} \leq C 2^{-j} .
$$

In conclusion, by $(6.2),(6.4),(6.5)$ we obtain

whence the result follows.

$$
\left\|\int_{|\xi| \geq \varepsilon} \widehat{\psi}(\xi-\eta) \frac{\eta}{|\eta|^{3}} d \eta\right\|_{L^{2}\left(2^{j-1} \leq|\xi| \leq 2^{j+1}\right)} \leq C \cdot 2^{-j}
$$

For our second Lemma we need an alternative expression of the Besov norm (see e.g. [15], §2.5.1): given $\delta>0$, which may be chosen arbitrarily,

$$
\|u\|_{B_{2, \infty}^{1}\left(\mathbb{R}^{2}\right)} \simeq\|u\|_{L^{2}}+\sup _{|h| \leq \delta}|h|^{-1}\left\|\Delta_{h}^{2} u\right\|_{L^{2}\left(\mathbb{R}^{2}\right)} .
$$

Here $\Delta_{h}^{2}$ is the second difference operator

$$
\Delta_{h}^{2} f(x)=f(x+2 h)-2 f(x+h)+f(x),
$$

while the first difference operator is simply

$$
\Delta_{h}^{1} f(x)=f(x+h)-f(x) .
$$


Lemma 6.2. Consider the function on $\mathbb{R}^{2}$

$$
\lambda(x)=\sqrt{1-|x|^{2}} \text { for }|x| \leq 1, \quad 0 \text { elsewhere, } .
$$

Then, given any $f(s, x) \in C^{2}\left(\mathbb{R}^{+} \times \mathbb{R}^{2}\right)$ with bounded derivatives, the composition $f(\lambda(x), x)$ belongs locally to the Besov space $B_{2, \infty}^{1}$, i.e., it belongs to $B_{2, \infty}^{1}(\Omega)$ for any bounded open set $\Omega \subseteq \mathbb{R}^{2}$. In particular, $\lambda(x)$ itself belongs to $B_{2, \infty}^{1}\left(\mathbb{R}^{2}\right)$.

Proof. Choose any cutoff function $\psi(x)$, and define

$$
g(x)=\psi(x) f\left(\sqrt{1-|x|^{2}}, x\right) .
$$

We need a suitable estimate for $\Delta_{h}^{2} g(x)$. Notice that, given any $C^{2}$ function $\Phi(y)$ on $\mathbb{R}^{N}$ and any locally bounded function $\gamma: \mathbb{R}^{n} \rightarrow \mathbb{R}^{N}$, the following formulas hold:

$$
\Delta_{h}^{1} \Phi(\gamma(x))=\left\langle a, \Delta_{h}^{1} \gamma(x)\right\rangle
$$

where $a=a(\gamma(x+h), \gamma(x))$, for a suitable $C^{1}$ vector valued function $a(y, z)$, and

$$
\Delta_{h}^{2} \Phi(\gamma(x))=\left\langle a, \Delta_{h}^{2} \gamma(x)\right\rangle+\left\langle A_{1} \Delta_{h}^{1} \gamma(x+h), \Delta_{h}^{1} \gamma(x)\right\rangle+\left\langle A_{2} \Delta_{h}^{1} \gamma(x), \Delta_{h}^{1} \gamma(x)\right\rangle,
$$

where $a=a(\gamma(x+h), \gamma(x)), A_{j}=A_{j}(\gamma(x+2 h), \gamma(x+h), \gamma(x))$, for $a(y, z)$, $A_{j}(y, z, p)$ continuous functions of their arguments (vector- and matrix- valued respectively). If we apply these formulas to $g(x)$, since $x, h$ run on a compact set we get

$$
\left|\Delta_{h}^{1} g(x)\right| \leq C\left|\Delta_{h}^{1} \lambda(x)\right|
$$

and

$$
\left|\Delta_{h}^{2} g(x)\right| \leq C\left|\Delta_{h}^{2} \lambda(x)\right|+C\left|\Delta_{h}^{1} \lambda(x)\right|^{2}+C\left|\Delta_{h}^{1} \lambda(x+h)\right|^{2}
$$

with $C$ independent of $x, h$. We are thus reduced to estimate the differences of $\lambda(x)$. For a fixed $|h| \leq 1 / 4$, we split $\mathbb{R}^{2}$ in the three domains $|x|>1+3|h|$, $1+3|h|>|x|>1-3|h|$ and $|x|<1-3|h|$. In the first one we have simply

$$
\Delta_{h}^{1} \lambda(x)=\Delta_{h}^{1} \lambda(x+h)=\Delta_{h}^{2} \lambda(x)=0 .
$$

In the region $1+3|h|>|x|>1-3|h|$ we have directly

$$
\left|\Delta_{h}^{1} \lambda(x)\right| \leq 6|h|^{1 / 2}, \quad\left|\Delta_{h}^{1} \lambda(x+h)\right| \leq 7|h|^{1 / 2}, \quad\left|\Delta_{h}^{2} \lambda(x)\right| \leq 16|h|^{1 / 2}
$$

and since the measure of the region is $12 \pi|h|$ we obtain for some constant independent of $h$

$$
\begin{gathered}
\left\|\left|\Delta_{h}^{1} \lambda(x)\right|+\left|\Delta_{h}^{1} \lambda(x+h)\right|\right\|_{L^{2}(1+3|h|>|x|>1-3|h|)} \leq C|h| . \\
\left\|\Delta_{h}^{2} \lambda\right\|_{L^{2}(1+3|h|>|x|>1-3|h|)} \leq C|h| .
\end{gathered}
$$

In the last region $|x|<1-3|h|$ some more computation is needed; notice that here $\lambda(x)=\sqrt{1-|x|^{2}}$. We have

$$
\Delta_{h}^{1} \sqrt{1-|x|^{2}}=-\frac{|h|^{2}+2 x \cdot h}{\sqrt{1-|x|^{2}}+\sqrt{1-|x+h|^{2}}}
$$

and this implies

$$
\left|\Delta_{h}^{1} \sqrt{1-|x|^{2}}\right| \leq 2|h|^{1 / 2}
$$

the inequality

$$
\left|\Delta_{h}^{1} \sqrt{1-|x+h|^{2}}\right| \leq 2|h|^{1 / 2}
$$

is analogous. Thus we have

$$
\|\left|\Delta_{h}^{1} \sqrt{1-|x|^{2}}\right|+\left.\left|\Delta_{h}^{1} \sqrt{1-|x+h|^{2}}\right|\right|_{L^{2}(|x|<1-3|h|)} \leq C|h|
$$


with $C$ independent of $h$. In a similar way, some elementary algebra gives

$$
\Delta_{h}^{2} \sqrt{1-|x|^{2}}=\frac{|h|^{2}}{m_{1}}-3 \frac{|h|^{2}}{m_{2}}-2 x \cdot h \frac{|h|^{2}+2 x \cdot h}{m_{1} \cdot m_{2} \cdot m_{3}},
$$

where we have introduced the quantities

$$
\begin{gathered}
m_{1}=\sqrt{1-|x|^{2}}+\sqrt{1-|x+h|^{2}}, \\
m_{2}=\sqrt{1-|x|^{2}}+\sqrt{1-|x+2 h|^{2}}, \\
m_{3}=\sqrt{1-|x+2 h|^{2}}+\sqrt{1-|x+h|^{2}} .
\end{gathered}
$$

Since $|x|<1-3|h|$, we have $m_{1} \geq 2|h|^{1 / 2}, m_{2} \geq 2|h|^{1 / 2}$, and this allows to estimate the first two terms in (6.12) as follows:

$$
\left|\frac{|h|^{2}}{m_{1}}-3 \frac{|h|^{2}}{m_{2}}\right| \leq 2|h|^{3 / 2} .
$$

On the other hand, $|x| \leq 1-3|h|$ implies

$$
2|x+h|^{2} \leq|x|^{2}+|x|^{2}+2|h|^{2}+4|x| \cdot|h| \leq|x|^{2}+1
$$

whence

and this implies

$$
2-2|x+h|^{2} \geq 1-|x|^{2}
$$

while it is obvious that

$$
m_{3} \geq \frac{1}{2} \sqrt{1-|x|^{2}}
$$

$$
m_{1} \geq \sqrt{1-|x|^{2}}, \quad m_{2} \geq \sqrt{1-|x|^{2}} .
$$

These inequalities give for the third term in (6.12)

$$
\left|2 x \cdot h \frac{|h|^{2}+2 x \cdot h}{m_{1} \cdot m_{2} \cdot m_{3}}\right| \leq \frac{12|h|^{2}}{\left(1-|x|^{2}\right)^{3 / 2}} .
$$

Summing up, we have obtained on the region $|x|<1-3|h|$ the inequality

$$
\left|\Delta_{h}^{2} \sqrt{1-|x|^{2}}\right| 2|h|^{3 / 2}+\frac{12|h|^{2}}{\left(1-|x|^{2}\right)^{3 / 2}}
$$

and integrating with respect to $x$ we obtain

$$
\left\|\Delta_{h}^{2} \sqrt{1-|x|^{2}}\right\|_{L^{2}(|x|<1-3|h|)} \leq C|h|
$$

with $C$ independent of $h$. In conclusion, (6.8), (6.9), (6.10), (6.11), (6.13) combined with (6.7) give

and this implies the thesis.

$$
\left\|\Delta_{h}^{2} g\right\|_{L^{2}\left(\mathbb{R}^{2}\right)} \leq C|h|
$$

The last lemma is useful to check whether the distributional derivative of a piecewise smooth function coincides with its derivative a.e.:

Lemma 6.3. Let $\Omega \subseteq \mathbb{R}^{n}$ be an open set and $K \subseteq \mathbb{R}^{n}$ be a closed set, and denote by $K_{\varepsilon}=\{x: d(x, K)<\varepsilon\}$ the $\varepsilon$-neighborhood of $K$. Let $f \in L_{\mathrm{loc}}^{1}(\Omega)$ be a function, differentiable a.e. on $\Omega \backslash K$ with differential $\nabla f$. Assume that

(i) $\nabla f$ is in $L_{\mathrm{loc}}^{1}(\Omega \backslash K)$ and coincides with the distributional derivative of $f$ on $\Omega \backslash K$;

(ii) there exists a smooth function $h(x)$ defined on a neighborhood of $K$ such that for any compact set $B \subseteq \Omega$

$$
\lim _{\varepsilon \downarrow 0} \frac{1}{\varepsilon} \int_{K_{\varepsilon} \cap B}|f-h| d x=0 .
$$


Then the distributional derivative of $f$ coincides with the function $g \in L_{\mathrm{loc}}^{1}(\Omega)$ defined as $\nabla f$ on $\Omega \backslash K$ and $\nabla h$ on $K$.

If in addition $K$ has measure 0 , then the distributional derivative and the derivative a.e. of $f$ on $\Omega$ coincide.

Proof. Let $\rho_{\varepsilon}$ be a sequence of standard mollifiers with support in $B(0, \varepsilon)$ and set

$$
\phi_{\varepsilon}=\rho_{\varepsilon} * \chi_{2 \varepsilon}
$$

where $\chi_{2 \varepsilon}$ is the characteristic function of the set $K_{2 \varepsilon}$. Notice that

$$
\phi_{\varepsilon}=1 \text { on } K_{\varepsilon}, \quad \operatorname{supp} \phi_{\varepsilon} \subseteq K_{4 \varepsilon} ;
$$

moreover, on any compact set $B$ we have the pointwise estimate

$$
\left|\nabla \phi_{\varepsilon}\right|=\left|\left(\nabla \rho_{\varepsilon}\right) * \chi_{2 \varepsilon}\right| \leq \frac{C(B)}{\varepsilon} .
$$

Now fix any test function $\phi$ with support in $\Omega$; we can write

$$
\begin{aligned}
-\int f \nabla \phi d x & =-\int f \nabla\left[\phi\left(1-\phi_{\varepsilon}\right)\right] d x-\int(f-h) \nabla\left[\phi \phi_{\varepsilon}\right] d x-\int h \nabla\left[\phi \phi_{\varepsilon}\right] d x= \\
& =I_{\varepsilon}+I I_{\varepsilon}+I I I_{\varepsilon} .
\end{aligned}
$$

By (i) we have immediately

$$
I_{\varepsilon}=\int \nabla f\left[\phi\left(1-\phi_{\varepsilon}\right)\right] d x \rightarrow \int_{\Omega \backslash K} g \phi d x
$$

as $\varepsilon \rightarrow 0$. Moreover we can write

$$
\left|I I_{\varepsilon}\right| \leq \frac{C}{\varepsilon} \int_{K_{4 \varepsilon} \cap B}|f-h| d x \rightarrow 0
$$

where $B$ is the support of $\phi$; we have used the pointwise estimate and assumption (ii). Finally, we have

$$
I I I_{\varepsilon}=\int \nabla h \phi \phi_{\varepsilon} d x \rightarrow \int_{K} \nabla h \phi d x=\int_{K} g \phi d x
$$

and summing up we obtain the thesis. The last claim follows immediately.

\section{REFERENCES}

[1] T. Cazenave, J. Shatah, and A. S. Tahvildar-Zadeh, Harmonic maps of the hyperbolic space and development of singularities in wave maps and Yang-Mills fields, Ann. Inst. $H$. Poincaré Phys. Théor. 68, 3 (1998), 315-349.

[2] Y. Choquet-Bruhat, Global existence for non-linear $\sigma$-models, Rend. Sem. Mat. Univ. Pol. Torino, Special Issue (1988), 65-86.

[3] D. Christodoulou and A. S. Tahvildar-Zadeh, On the regularity of spherically symmetric wave maps, Comm. Pure Appl. Math. 46, 7 (1993), 1041-1091.

[4] P. D'Ancona, V. Georgiev On the continuity of the solution operator to the wave map system Preprint Università di Pisa, No.2.439.1404 (july 2002)

[5] J. Ginibre And G. Velo, The Cauchy problem for the o $(N), \operatorname{cp}(N-1)$, and $G_{\mathbf{C}}(N, p)$ models, Ann. Physics 142, 2 (1982), 393-415.

[6] M. G. Grillakis, The wave map problem, In Current developments in mathematics, 1997 (Cambridge, MA). Int. Press, Boston, MA, 1999, pp. 227-230.

[7] C. H. Gu, On the Cauchy problem for harmonic maps defined on two-dimensional Minkowski space, Comm. Pure Appl. Math. 33, 6 (1980), 727-737.

[8] S. KLAinerman And M. Machedon, On the regularity properties of a model problem related to wave maps, Duke Math. J. 87, 3 (1997), 553-589.

[9] S. Klainerman and S. Selberg, Remark on the optimal regularity for equations of wave maps type, Comm. Partial Differential Equations 22, 5-6 (1997), 901-918.

[10] S. Müller And M. Struwe, Global existence of wave maps in $1+2$ dimensions with finite energy data, Topol. Methods Nonlinear Anal. 7, 2 (1996), 245-259.

[11] K. Nakanishi, M. Oнta, On global existence of solutions to nonlinear wave equations of wave map type, Nonlinear Anal. TMA 42, (2000), 1231-1252. 
[12] J. Shatah, Weak solutions and development of singularities of the su(2) $\sigma$-model, Comm. Pure Appl. Math. 41, 4 (1988), 459-469.

[13] J. Shatah And M. Struwe, Geometric wave equations, New York University Courant Institute of Mathematical Sciences, New York, 1998.

[14] J. Shatah and A. S. Tahvildar-Zadeh, On the Cauchy problem for equivariant wave maps, Comm. Pure Appl. Math. 47, 5 (1994), 719-754.

[15] E. M. Stein And G. Weiss, Introduction to Fourier analysis on Euclidean spaces, Princeton University Press, Princeton, N.J., 1971, Princeton Mathematical Series, No. 32.

[16] M. Struwe, Wave maps, In Nonlinear partial differential equations in geometry and physics (Knoxville, TN, 1995). Birkhäuser, Basel, 1997, pp. 113-153.

[17] T. TAO, Global regularity of wave maps II. Small energy in two dimensions, Comm. Math. Phys. 224, (2001), 443-544.

[18] D. TAtaru, On global existence and scattering for the wave maps equation, Amer. J. Math. 123, 1 (2001), 37-77.

[19] H. TRIEBEL, Interpolation theory, function spaces, differential operators, second ed., Johann Ambrosius Barth, Heidelberg, 1995.

[20] Y. ZHou, Global weak solutions for $(1+2)$-dimensional wave maps into homogeneous spaces, Ann. Inst. H. Poincaré Anal. Non Linéaire 16, 4 (1999), 411-422.

Piero D'Ancona: Unversità di Roma "La Sapienza", Dipartimento di Matematica, PiAZZAle A. Moro 2, I-00185 Roma, ItAly

E-mail address: dancona@mat.uniroma1.it

Vladimir Georgiev: Università di Pisa, Dipartimento di Matematica, Via Buonarroti 2, I-56127 Pisa, ITALY

E-mail address: georgiev@dm.unipi.it 